\title{
Capturing preferences for inequality aversion in decision support
}

\author{
Özlem Karsu ${ }^{\mathrm{a}, *}$, Alec Morton $^{\mathrm{b}}$, Nikos Argyris ${ }^{\mathrm{c}}$ \\ a Department of Industrial Engineering, Bilkent University, Ankara, Turkey \\ ${ }^{\mathrm{b}}$ Management Science Department, University of Strathclyde Business School, Glasgow, UK \\ ' School of Business and Economics, Loughborough University, Leicestershire, UK
}

\section{A R T I C L E I N F O}

\section{Article history:}

Received 12 December 2016

Accepted 1 July 2017

Available online 10 July 2017

\section{Keywords:}

Multiple criteria analysis

Equitable preferences

Generalised Lorenz dominance

Conditional dominance

Interactive approaches

\begin{abstract}
A B S T R A C T
We investigate the situation where there is interest in ranking distributions (of income, of wealth, of health, of service levels) across a population, in which individuals are considered preferentially indistinguishable and where there is some limited information about social preferences. We use a natural dominance relation, generalised Lorenz dominance, used in welfare comparisons in economic theory. In some settings there may be additional information about preferences (for example, if there is policy statement that one distribution is preferred to another) and any dominance relation should respect such preferences. However, characterising this sort of conditional dominance relation (specifically, dominance with respect to the set of all symmetric increasing quasiconcave functions in line with given preference information) turns out to be computationally challenging. This challenge comes about because, through the assumption of symmetry, any one preference statement ("I prefer giving $\$ 100$ to Jane and $\$ 110$ to John over giving $\$ 150$ to Jane and $\$ 90$ to John") implies a large number of other preference statements ("I prefer giving $\$ 110$ to Jane and $\$ 100$ to John over giving $\$ 150$ to Jane and $\$ 90$ to John"; "I prefer giving $\$ 100$ to Jane and $\$ 110$ to John over giving $\$ 90$ to Jane and $\$ 150$ to John"). We present theoretical results that help deal with these challenges and present tractable linear programming formulations for testing whether dominance holds between any given pair of distributions. We also propose an interactive decision support procedure for ranking a given set of distributions and demonstrate its performance through computational testing.
\end{abstract}

(c) 2017 Elsevier B.V. All rights reserved.

\section{Introduction}

\subsection{The problem of fair allocation and application domains}

An overriding concern in matters of public (and sometimes private) sector management is the equitability in the distribution of good (or alternatively bad) outcomes (income, wealth, health, service quality) across persons or population groups flowing from some particular decision. This might apply, for example, where budgetary pressures make it imperative to reform taxation or welfare arrangements; where differences in facility location lead to variations in accessibility (travelling times to nearest hospital, speed of internet service provision); or where social policies to redress the plight of deprived populations (provision of recreational facilities, early life educational interventions) are being contemplated.

\footnotetext{
* Corresponding author.

E-mail addresses: ozlemkarsu@bilkent.edu.tr, ozlemkarsu@yahoo.co.uk

(Ö. Karsu), alec.morton@strath.ac.uk (A. Morton), n.argyris@lboro.ac.uk (N. Argyris).
}

There is a large body of literature on applications where equity concerns naturally arise and are considered (reviewed in Karsu \& Morton, 2015). Note that in most of these applications, equity is rarely the sole concern and the decision makers consider both fairness and efficiency as important. However, they generally find it difficult to explicitly state the equity-efficiency tradeoffs which underlie their decisions (due to a number of reasons elaborated in the ensuing discussion). In this study we consider such problems and try to support the decision makers in these settings by helping them choose their most preferred solution or rank the solutions based on preference. Some examples of the applications in which equity concerns arise are the following:

- Bandwidth allocation on a network: The problem is making bandwidth allocations considering equity over users as well as efficiency (throughput) (Luss, 2010; Ogryczak, Wierzbicki, and Milewski, 2008).

- Public service facility location: Locating a public service facility involves equity concerns over the customers as well as efficiency concerns (e.g. minimising total distance travelled to the facility) (Ogryczak, 1999; Ogryczak, 2009). 
- Capital-budgeting with fairness concerns: Health care project funding settings, in which project portfolios are evaluated based on the distribution of the potential benefit to different population groups are an example (Morton, 2014). Trying to achieve more equitable investments across different sectors resulting from an underlying motive for risk reduction is another example relevant in many settings (Karsu \& Morton, 2014).

- Ranking countries with respect to social welfare: Comparing income distributions of different countries (Shorrocks, 1983).

- Workload allocation: Managers have concerns for ensuring an equitable workload allocation when assigning tasks to staff.

Taking the workload allocation example, a motivating case is a problem faced by a firm working in the heating ventilation and air conditioning (HVAC) sector in Turkey (Karsu and AzizogĨu, 2012; Karsu \& Azizoglu, 2014). The manager faces the problem of assigning staff (agents) to tasks such that once assigned the agent will perform the task for multiple periods. Agents have different levels of experience across different types of tasks, hence the time required to perform a task is different for different agents. Each feasible assignment results in a load distribution over the agents.

An assignment that minimises the total workload, which may be considered as the most efficient solution, in fact may result in an extremely unfair workload allocation across the agents. On the other hand, the (lexicographic) max-min fairness solution (which first minimises the maximum workload over all the agents, then the second maximum and so on), which is sometimes referred to as the most equitable solution (Luss, 2012), may significantly increase the total workload. Therefore, there is usually a need to seek compromise solutions between these two extreme solutions.

Although managers may concur that both fairness and efficiency are important in workload allocation, in our experience they are generally unable to articulate a precise mathematical expression which can serve as an objective function balancing these two competing objectives, or explicitly state the equity-efficiency tradeoffs which underlie their decisions. This may be for political reasons-stakeholders may be unhappy to learn precisely, quantitatively, how much their service providers or elected representatives care about them relative to others-or may reflect genuine cognitive difficulties, as assessing relative desert is an unstructured task and may involve balancing multiple conflicting considerations. As an example, consider the following two allocations of a good across three indistinguishable entities: $(2,7,8)$ and $(4.5,4.5,8)$. Common sense suggests that the second distribution is better since the total amount of 17 units is more equitably allocated. Now consider $(2,7,8)$ and $(4,4,5)$. The first allocation seems more efficient in the sense that the total amount allocated is larger but in the second one the good seems more equitably distributed. We observe the trade-off between equity and efficiency here. In this example there is no "objective" way of choosing the better allocation and different people may take different views.

The problems we mention above can be thought of as what Karsu and Morton (2015) call equitability problems. In this work, we consider such equitability problems in which a social planner (henceforth "SP") who has equity concerns as well efficiency concerns tries to compare distributions of a good over multiple parties, when there is symmetry (meaning that the identities of the parties are not important and do not affect the decision, hence Jane getting $\$ 100$ and John getting $\$ 150$ is as good as John getting \$100 and Jane getting \$150 for the SP). As mentioned before, in general SPs may be unable to specify a mathematical expression that encapsulates the trade-off between efficiency and equity; yet in many settings it is possible to obtain social preferences involving distributions (for example by asking the SP "Do you prefer distribution $a$ or distribution $b$ ?").
This leads naturally to the question which is precisely the problem we study in this work: How do we support a SP who is confronted with a set of distributions to select the best one or rank them from the most preferred to the least, taking into account available information about social preferences (for example the direct expression by the Minister that one distribution is to be preferred to another distribution)? As we demonstrate later in Section 3, the classical results in the literature on equitability problems (see Karsu and Morton, 2015 for a review) are not suited to the problem of comparing distributions while taking preferences into account. In this paper we propose a new method for this problem which is based on the use of such information to infer more about the SP's preferences. Specifically, preference information will help us to refine the ranking of the distributions in the sense that given one distribution $a$ is preferred to another distribution $b$ by the SP, we will be able to make statements such as "she should also prefer distribution $c$ to $d$ ", even if the SP does not express a preference relation over $c$ and $d$ directly. Checking whether such statements can be made between any given pair of distributions requires calculations of combinatorial complexity due to symmetry. We introduce substantial theory to tackle the technical challenges due to the symmetry property of such problems as we will discuss in Section 4.

Our contributions in this paper can be summarised as follows:

- We present an effective way of using preference information in equitability problems. We discuss how preferences can be used to derive a stronger ordering of distributions under consideration.

- We introduce results to address the significant computational problems of deriving the stronger ordering of distributions. Specifically, by Theorems 5 and 6 in Section 4, we address the intractability problem due to symmetry.

- We illustrate the implementability of our approach by applying it to a ranking problematique, i.e. the category of decision problems consisting of the effort to rank the distributions from the best to the worst, which arises naturally in many settings.

\section{Overview of related work}

There is a broad literature in economics dealing with equity (see e.g. Sen and Foster, 1997; Young, 1994). In this section we provide a review of the most relevant work on comparing distributions and clarify our contribution to the literature.

\subsection{The theory of majorisation}

The theory of majorisation gives a frame for comparing distributions in the absence of preference information (Hardy, Littlewood, and Polya, 1934; Marshall, Olkin, and Arnold, 2009; Müller and Stoyan, 2002; Shaked \& Shanthikumar, 2007). We sketch this well-known theory below.

Let $Z$ denote a (finite) set of distributions (alternatives) with a typical member as $z^{i}=\left(z_{1}^{i}, \ldots, z_{p}^{i}\right)$, where $z^{i} \in Z$ represents a distribution of a good among $p$ parties and hence $z_{j}^{i}$ is the amount of good that party $j$ gets in distribution $i$. Given $z \in \mathbb{R}^{p}\left(\mathbb{R}^{p}\right.$ denotes the $n$-dimensional real space), let $\vec{z}$ denote the ordered permutation of $z$ such that $\vec{z}_{1} \leq \vec{z}_{2} \leq \ldots \leq \vec{z}_{p}$.

A distribution $z^{1} \in \mathbb{R}^{p}$ is majorised by another distribution $z^{2} \in$ $\mathbb{R}^{p}$ if $\sum_{j=1}^{p} \overrightarrow{z_{j}^{1}}=\sum_{j=1}^{p} \overrightarrow{z_{j}^{2}}$ (i.e. they have the same total output) and $\sum_{j=1}^{i} \overrightarrow{z_{j}^{1}} \geq \sum_{j=1}^{i} \overrightarrow{z_{j}^{2}} \forall i<p$. An application of majorisation is in comparing distributions with respect to inequality and the resulting quasi-order is called Lorenz order (introduced by Lorenz (1905)) in the economics literature. If $z^{1}$ is majorised by $z^{2}$ then it represents a more equitable allocation of the same amount of output to the parties, i.e. it Lorenz dominates $z^{2}$. 


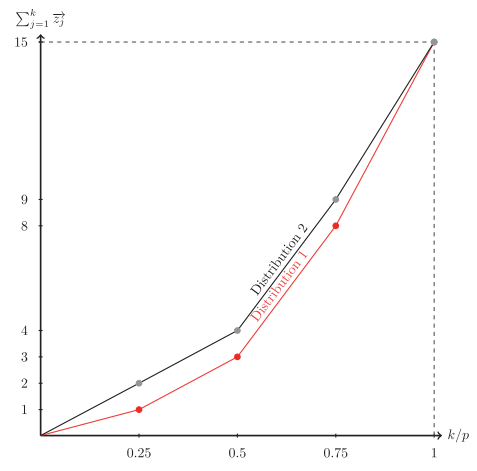

(a) GL 1

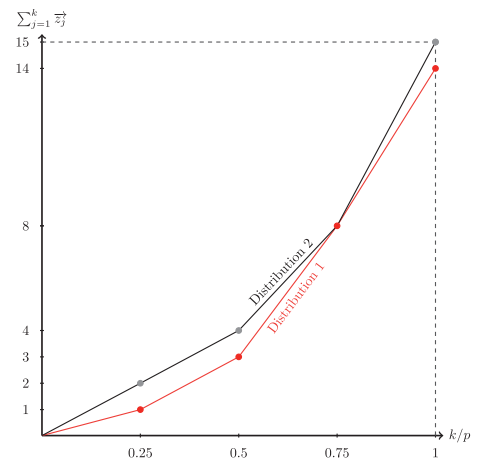

(b) GL 2

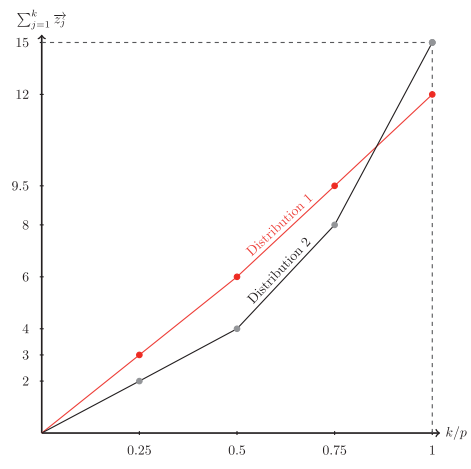

(c) GL 3

Fig. 1. Generalised Lorenz curves.

Note that majorisation and hence Lorenz dominance order allow us to distinguish distributions with the same amount of total output. An extension of these concepts to distributions with different total outputs is the generalised Lorenz order, introduced by Shorrocks (1983).

Definition 1. A distribution $z^{2}$ generalised Lorenz dominates another distribution $z^{1}$ (denoted by $z^{1} \preceq_{G L} \quad z^{2}$ ) if $\sum_{j=1}^{i} \overrightarrow{z_{j}^{1}} \leq$ $\sum_{j=1}^{i} \overrightarrow{z_{j}^{2}} \forall i$.

Starting from the origin (0) and plotting the points $\left(\sum_{j=1}^{k} \overrightarrow{z_{j}}, k / p\right)$ for all $k=1, \ldots, p$ and joining these points by line segments provides the so called generalised Lorenz curve of a distribution $z$. Hence, $z^{1} \preceq_{G L} z^{2}$ if the corresponding generalised Lorenz curve does not lie below that of the latter. Generalised Lorenz dominance is referred to as equitable dominance in the multicriteria decision making literature (Baatar and Wiecek, 2006; Kostreva and Ogryczak, 1999; Kostreva, Ogryczak, \& Wierzbicki, 2004; Mut \& Wiecek, 2011; Ogryczak, 2000).

Example 1. Consider the following distribution pairs: $(1,2,5,7)$ and $(2,2,5,6) ;(1,2,5,6)$ and $(2,2,4,7) ;(3,3,3.5,3.5)$ and $(2,2,4,7)$. The generalised Lorenz curves of these distribution pairs are seen in Fig. $1 \mathrm{a}, \mathrm{b}$ and $\mathrm{c}$ respectively. In Fig. $1 \mathrm{a}$ and $\mathrm{b}$ the second distribution dominates the first one and in Fig. 1c neither of the distributions dominates the other. In the first example, the same amount of total output is distributed more equitably in the second distribution (in $(1,2,5,7)$, taking 1 unit of output from the richest entity and giving it to the worst off one results in the second distribution $(2,2,5,6)$, such an equity-enhancing transfer leads to a better distribution). In the second example, the amount allocated to the $k$ poorest entities in the second distribution is always at least as high as the amount allocated in the first distribution for all $k=1,2, \ldots, p$. In the third example, distribution 1 is more equitable but there is greater total wealth in distribution 2, and hence we observe the trade-off between equity and efficiency.

Theorem 1 (Dasgupta, Sen, and Starrett, 1973; Rothschild and Stiglitz, 1973; Shorrocks, 1983). For any two distributions $z^{1}$ and $z^{2}$, $z^{1} \preceq_{G L} z^{2} \Leftrightarrow u\left(z^{1}\right) \leq u\left(z^{2}\right) \forall u(.) \in Q_{\text {sym }}$, where $Q_{\text {sym }}$ is the set of symmetric increasing strictly quasiconcave ${ }^{1}$ social evaluation functions.

\footnotetext{
${ }^{1}$ A function $u($.$) is strictly quasiconcave if for all z^{1}, z^{2}: z^{1} \neq z^{2}$ and $\alpha \in(0,1)$ we have $u\left(\alpha z^{1}+(1-\alpha) z^{2}\right)>\min \left\{u\left(z^{1}\right), u\left(z^{2}\right)\right\}$. A function $u($.$) is symmetric if for all$ $z \in \mathbb{R}^{p}, u(z)=u\left(\Pi^{s}(z)\right)$ for all $s=1, \ldots, p$ !, where $\Pi^{s}(z)$ is an arbitrary permutation of $z$. In other words, the function assigns the same value to all permutations of a distribution.
}

By Theorem 1, another way to look at generalised Lorenz dominance is dominance with respect to the set of symmetric increasing strictly quasiconcave social evaluation functions.

\subsection{Second order stochastic dominance}

Another frame to consider while comparing distributions in the absence of preference information is the second order stochastic dominance (SSD), which concerns itself with comparing distributions of risky options (Müller and Stoyan, 2002; Shaked \& Shanthikumar, 2007). The analogy between stochastic dominance and inequality comparisons is well-established in the literature. The classic works in the theory of inequality measurement draw on the analogy between inequality- and risk- aversion (Atkinson, 1970). To underscore the qualitative analogy, one way to think about the comparison of societies $a$ and $b$ with different distributions of income is to ask oneself the question: "If I was to wake up tomorrow with the life circumstances of a randomly chosen individual, would I prefer to be a randomly chosen member of society $a$ or society $b$ ?".

In the context of inequality comparisons SSD can be defined as follows:

Definition 2. A distribution $z^{2}$ dominates another distribution $z^{1}$ in the sense of second order stochastic dominance (denoted $z^{1} \preceq S S D$ $\left.z^{2}\right)$ if $u\left(z^{1}\right) \leq u\left(z^{2}\right)$ for all social evaluation functions of the form $u()=.\sum_{j} v\left(z_{j}\right)$, where $v($.$) is concave (Levy, 1992). { }^{2}$

It is worth noting here that the problems of comparison of risks and the comparison of income distributions are not precisely formally identical. The main mathematical difference is that, when comparing risky options, utility (evaluation) functions are generally taken to be additively separable over states (reflecting the "sure thing principle" that one's preferences for consequences which one does experience should not depend on the consequences in the states of nature which are not realised) (Hurley, 1992; Jeffrey, 1982; Savage, 1954; Wakker, 1989). In the theory of inequality there is no compelling argument for separability in the same way, and indeed some writers argued that a theory of inequality should take into account "caring externalities" , that is to say, the distress A feels at B's disadvantage (Culyer, 1989, see also Diamond, 1967 for a criticism of assuming the sure thing principle for social choice). The need to take into account such caring externalities provides a compelling argument that, in the inequality context,

\footnotetext{
2 To be more precise, SSD is defined by using expectations, i.e. $\Sigma_{i} p_{i} u\left(z_{i}\right)$. However, this would be equivalent to the definition we use above. To see this, observe that the expectation can be obtained by the additive aggregation (and vice versa) by dividing by $n$, the number of parties, and factorising.
} 
unlike the risk context, a comprehensive theory has to allow for the possibility of nonadditivity in the evaluation function. Moreover, several natural evaluation functions have a non-additive form. For example, think about a situation where we evaluate alternative monetary allocations to two people A and B, who are otherwise indistinguishable. Suppose that giving \$1 more to A (B) is worth 1 util to the social planner as long as the difference between what A (B) already has and what B (A) already has is less than a certain threshold, say $\$ 10$. When the difference $(A-B)((B-A))$ exceeds that threshold every $\$ 1$ added to the income of $A(B)$ is worth 0.5 utils to the social planner. Such a preference statement cannot apply to a social planner with an additive evaluation function.

It is well known that checking dominance with respect to the functions of the form $u()=.\sum_{j} v\left(z_{j}\right)$, where $v($.$) is concave, is$ equivalent to checking dominance with respect to the set of functions that are symmetric, increasing and strictly quasi-concave ( $Q_{\text {sym }}$ for short) in the absence of preference information (see Gravel and Moyes, 2013; Rothschild and Stiglitz, 1973; Shorrocks, 1983; Thistle, 1989).

Theorem 2. For any two distributions $z^{1}$ and $z^{2}, z^{1} \preceq_{S S D} z^{2} \Leftrightarrow z^{1} \preceq_{G L}$ $z^{2}$.

We commented previously that SPs may have preferences represented by non-additive evaluation functions. Yet, Theorem 2 illustrates that it would make no difference to merely include such functions to the set relative to which dominance is derived. In this sense, the results of Theorem 2 would be of little use to a SP who has already expressed preferences incompatible with an additive evaluation function (e.g. as in the previous example). In the following section we examine how this equivalence breaks when preferences expressed by SPs are taken into account.

\section{The usefulness of preference information: conditional dominance}

We now discuss the implications and usefulness of introducing preference information in comparing distributions of a good among a set of entities. We also demonstrate that this approach is both useful and substantively different to the existing approaches discussed in the previous section.

Consider a situation where a SP has provided preference information over a finite set $R$ of distributions, denoted $\preceq_{R}$. This could be a set of binary preference statements, or in the form of determining the least preferred distribution in a set of given reference distributions. Let $A\left(\unlhd_{R}\right)$ denote the set of additive functions of the form $u()=.\sum_{j} v\left(z_{j}\right)$, with $v($.) concave, that are compatible with the preference information $\preceq_{R}$. Specifically, for any two distributions one of which is preferred to the other by the SP, the compatible function $u($.$) has a higher value for the more preferred one.$ Similarly, let $Q_{s y m}\left(\varsigma_{R}\right)$ denote the set of strictly quasiconcave symmetric increasing functions that are compatible with $\preceq_{R}$. The sets $A\left(\varsigma_{R}\right)$ and $Q_{s y m}\left(\varsigma_{R}\right)$ are subsets of sets $A$ and $Q_{s y m}$ respectively. We can use these subsets to make further inferences about the social planners' preferences (choices) compared to the case where the original sets of functions $A$ or $Q_{s y m}$ are used.

Theorem 2 asserts that dominance with respect to sets $A$ and $Q_{\text {sym }}$ are equivalent, implying that additivity of the social evaluation function is not a material assumption in the absence of preference information. However, in the presence of preference information, additivity is a material assumption because dominance with respect to sets $A\left(\preceq_{R}\right)$ and $Q_{s y m}\left(\preceq_{R}\right)$ are not equivalent.

To see this, consider the following example.

Example 2. Table 1 shows four feasible allocations of a good over three people.
Table 1

\begin{tabular}{llll}
\multicolumn{3}{l}{ Example allocations of a good. } \\
\hline Alternative & Person 1 & Person 2 & Person 3 \\
\hline 1 & 2 & 7 & 8 \\
2 & 3 & 4 & 8 \\
3 & 2 & 7 & 1 \\
4 & 3 & 4 & 1
\end{tabular}

Suppose the SP compares between $(2,7,8)$ and $(3,4,8)$ and she prefers $(2,7,8)$. If we check dominance with respect to $A\left(\preceq_{R}\right)$ then we conclude that she must also prefer, for example $(2,7,1)$ to $(3,4,1)$ since if her preferences are represented by an additive function $u\left(z_{1}\right)+u\left(z_{2}\right)+u\left(z_{3}\right)$ then we have learned from her preference statements that $u(2)+u(7)>u(3)+u(4)$. Her preference for $(2,7,1)$ over $(3,4,1)$ depends on whether $u(2)+u(7)+u(1)$ is greater than or less than $u(3)+u(4)+u(1)$ but this is fixed by the above inequality. This means that all functions in $A\left(\preceq_{R}\right)$ would render distribution $(2,7,1)$ superior to distribution $(3,4,1)$. But this is not true if we allow general symmetric quasi-concave functions. For example, if the evaluation function $f$ is the sum of the pairwise minima, then $f(2,7,8)=\min (2,7)+\min (2,8)+$ $\min (7,8)=2+2+7=11$, and similarly, $f(3,4,8)=3+3+4=$ 10 but $f(2,7,1)=1+1+2=4$ and $f(3,4,1)=1+1+3=5$.

It is possible to check dominance with respect to $A\left(\preceq_{R}\right)$ using linear programming models and this is considered in some studies (see e.g. Armbruster and Delage, 2015; Karsu, 2016). However, to our knowledge, the problem of checking dominance with respect to $Q_{s y m}\left(\preceq_{R}\right)$ has not been tackled in the literature so far.

Our approach introduces this machinery to work with preference information and is able to accommodate the aforementioned social planner. But there is no a-priori insistence that the social planner's evaluation function is non-additive. Thus our approach can accommodate any social planner whose preferences are representable by functions in the set $Q_{s y m}\left(\varsigma_{R}\right)$ (of which $A\left(\varsigma_{R}\right)$ is a potentially empty subset). In sum, the approach verifies dominance with respect to the set $Q_{s y m}\left(\preceq_{R}\right)$ and extends generalised Lorenz dominance, which does not include any preference information, by incorporating preference information.

We shall use the term conditional generalised Lorenz dominance (c-dominance, denoted as $\preceq_{G L_{c}}$ ) to refer to dominance with respect to all social evaluation functions that are increasing, symmetric, strictly quasiconcave and consistent with $\preceq_{R}$.

Definition 3. Let $Q_{s y m}\left(\varsigma_{R}\right)$ be the set of increasing symmetric strictly quasiconcave social evaluation functions, which are compatible with some preference statement $\preceq_{R}$. Then for any two distributions $z^{1}$ and $z^{2}, z^{1} \preceq_{G L_{c}} z^{2} \Leftrightarrow u\left(z^{1}\right) \leq u\left(z^{2}\right) \forall u(.) \in Q_{\text {sym }}\left(\preceq_{R}\right)$.

Incorporating preference information and hence using conditional generalised Lorenz dominance would be useful as generalised Lorenz dominance cannot be used to compare even quite extreme distributions (consider e.g. $(3,4,7,11)$ and $(2,100,110$, 140). These two distributions do not generalised Lorenz dominate each other). Preference information can help us to compare two distributions $z^{1}$ and $z^{2}$ which are otherwise incomparable by generalised Lorenz dominance even if the SP has not expressed a preference relation over $z^{1}$ and $z^{2}$ directly. Thus, preference information can help refine the ranking of distributions under consideration. We demonstrate with an example.

Example 3. Suppose that the SP is considering a set of distributions of e.g. wealth over two people, seen in Table 2. In this set, the only pair that is comparable by the generalised Lorenz dominance relation is $z^{2}$ and $z^{3} ; z^{2}$ generalised Lorenz dominates $z^{3}$.

Now suppose that the SP provided the preference information that she prefers $z^{3}$ to $z^{1}$. What can we infer about the preference 


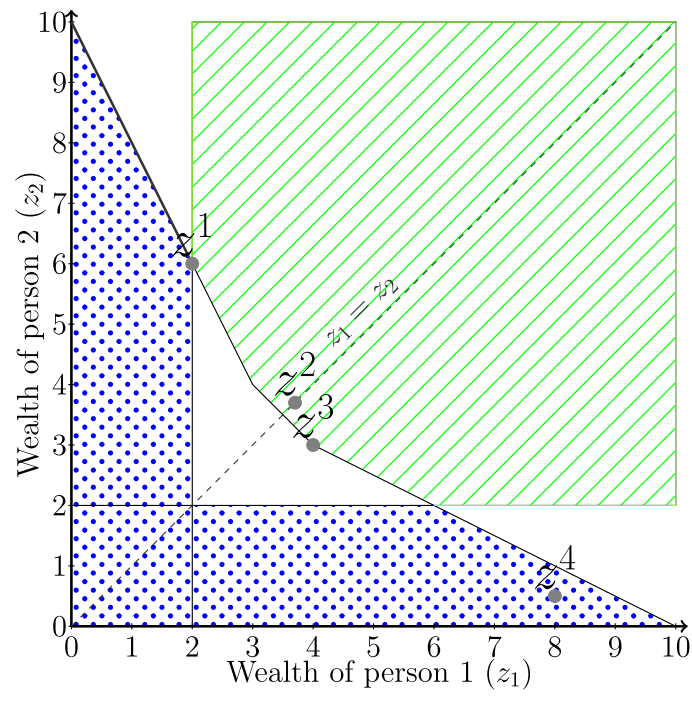

(a) Regions preferred to and less preferred than $z^{1}$ based on preference information $z^{1} \prec z^{3}$

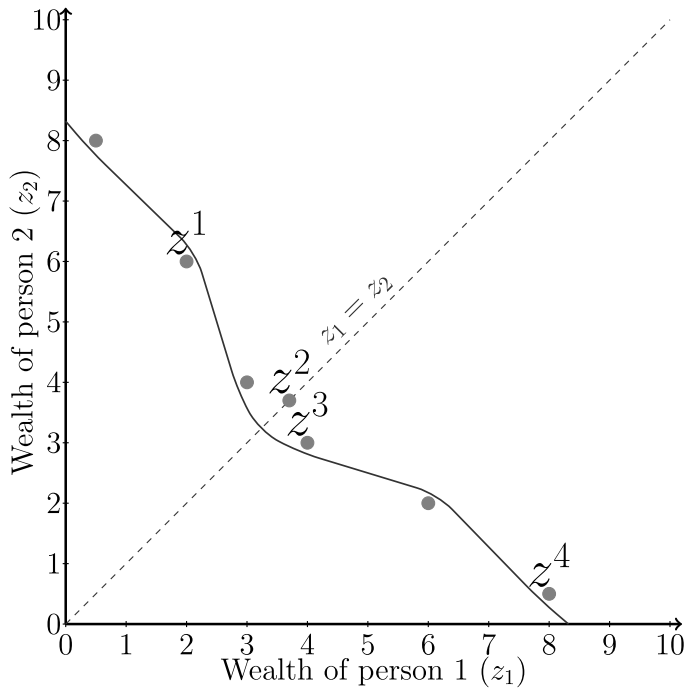

(b) A non-convex indifference curve

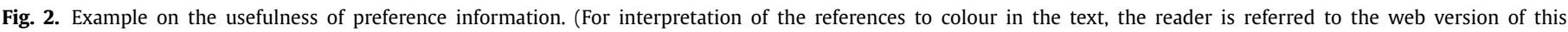
article.)

Table 2

Example distribution of wealth over two people.

\begin{tabular}{lll}
\hline Distribution & Person 1 & Person 2 \\
\hline$z^{1}$ & 2 & 6 \\
$z^{2}$ & 3.7 & 3.7 \\
$z^{3}$ & 4 & 3 \\
$z^{4}$ & 8 & 0.5 \\
\hline
\end{tabular}

relation between the distributions in this set by assuming that her social evaluation function is in set $Q_{s y m}$ ?

We can infer that $z^{2}$ should also be preferred to $z^{1}$ due to transitivity but this is trivial. What else can we infer from this preference statement?

Given $z^{3}$ is preferred to $z^{1}$, one can infer that for any decision maker with a symmetric increasing strictly quasiconcave function, the points in the blue dotted region in Fig. 2a will be less preferred than $z^{1}$, and the points that are in the green region with diagonal lines will be more preferred to $z^{1}$. We know this from quasiconcavity (i.e. the indifference curves are convex) and from symmetry. To see how; consider $z^{4}$, which is a point which should be less preferred than $z^{1}$. Suppose, on the contrary, that $z^{3}$ is preferred to $z^{1}$ but $z^{4}$ is also preferred to $z^{1}$. Then there has to be an indifference curve which separates $z^{3}$ and $z^{4}$ from $z^{1}$, with $z^{3}$ and $z^{4}$ (and all their permutations) above the indifference curve and $z^{1}$ (and its permutations) below the indifference curve (see Fig. $2 b$ for an example). But such a curve is not and cannot be convex, contradicting the assumption of quasiconcavity. So we can infer that $z^{1}$ is preferred to $z^{4}$ given $z^{3}$ is preferred to $z^{1}$. And the same reasoning goes for all points in the blue dotted region in Fig. 2a. Similarly, we can infer that the points in the green region with diagonal lines should be preferred to $z^{1}$.

\section{Theoretical results}

Table 3 summarises the notation and terminology used throughout the paper.
In this section we introduce results that allow for using preference information provided by a SP to refine the ranking of distributions. As seen in the example of the previous section, the preferences provided by the SP define, for each distribution, a nontrivial upper set, denoted $U(z)$ (the green etched region in Fig. 2a), and a non-trivial lower set, denoted $L(z)$ (the blue dotted region in Fig. 2a). In a setting where the SP's preferences are not characterised by symmetry, there are results in the literature that allow for characterising these sets. Such settings are discussed by Korhonen, Wallenius, and Zionts (1984) and Hazen (1983) (see Karsu, 2013 for more information). Relying on these results, however, is not possible where symmetry is a feature of the SP's preferences. In particular, symmetry would necessitate checking a set of conditions with respect to every possible combination of all permutations of a set of distributions, thus imposing an intractable computational load. Instead, our results provide a compact characterisation of $U(z)$ and $L(z)$ which avoid the need for considering all permutational checks, and in some cases avoid them altogether, thus affording tractability.

In the ensuing we will assume that the SP has provided preference information $\preceq_{R}$ of the form $z^{i} \succ z^{k}, i=1, \ldots, k-1$, which stands for "the SP prefers distribution $z^{i}$ to $z^{k}$ ". (Note that when $k=2$ this is pairwise comparison information). We will also use $R=\left\{z^{1}, \ldots, z^{k-1}\right\}$ to denote the set of reference distributions $z^{i}$ which the SP prefers to $z^{k}$. In practice the SP may provide further preferences (in the form of a partial ranking), but our assumption here does not restrict generality.

With use of the reference distributions in $R$, we also define a cone $C\left(R ; z^{k}\right)$ and a polyhedron $P\left(R ; z^{k}\right)$ as follows:

$$
\begin{aligned}
& C\left(R ; z^{k}\right)=\left\{z \mid z=z^{k}+\sum_{i=1}^{k-1} \mu_{i}\left(z^{k}-z^{i}\right), \mu_{i} \geq 0\right\}, \\
& P\left(R ; z^{k}\right)=\left\{z \mid z=\sum_{i=1}^{k} \mu_{i} z^{i}, \sum \mu_{i}=1, \mu_{i} \geq 0\right\},
\end{aligned}
$$

where the reference distributions $z^{i} \in R$ are referred to as the upper generators of the cone (and polyhedron) and $z^{k}$ as the lower generator. We express upper and lower sets for a distribution $z^{k}$ through cones and polyhedrons as follows.

$$
L_{\text {asym }}\left(z^{k}\right)=\left\{z \mid z \leq z^{\prime} \text { for some } z^{\prime} \in C\left(R ; z^{k}\right)\right\}
$$


Table 3

\begin{tabular}{|c|c|c|}
\hline Notation & Definition & Expression \\
\hline$z \in \mathbb{R}^{p}$ or $z^{i} \in \mathbb{R}^{p}$ & A generic alternative (distribution vector) & \\
\hline$Z$ & A (finite) set of distributions (alternatives) & \\
\hline$\Pi^{l}(z)$ & An arbitrary permutation of $z$ & \\
\hline$\vec{z}$ & Ordered vector of $z$ & $\vec{z}=\Pi^{l}(z): \vec{z}_{1} \leq \vec{z}_{2} \leq \ldots \leq \vec{z}_{p}$ \\
\hline$\preceq|<| \sim$ & Weak /strict/ indifference preference relation & \\
\hline $\bar{Q}$ & The set of functions that are increasing and strictly quasiconcave & \\
\hline$R$ & Set of distributions that are preferred to the same distribution $z^{k}$ & $R=\left\{z^{1}, z^{2}, \ldots, z^{k-1}\right\}$ \\
\hline$\preceq_{R}$ & Preference information taken from the SP & $z^{1}, \ldots, z^{k} \in \mathbb{R}^{p}$ such that $z^{i} \succ z^{k}$ \\
\hline$\preceq_{c}$ & Dominance with respect to $Q\left(\preceq_{R}\right)$ & Conditional dominance in the asymmetric case \\
\hline$Q_{s y m}$ & $\begin{array}{l}\text { The set of functions that are } \\
\text { strictly quasiconcave, symmetric and increasing }\end{array}$ & \\
\hline$\preceq_{G L}$ & Generalised Lorenz dominance relation & $\begin{array}{l}\text { Dominance without any preference information, } \\
\text { i.e. dominance with respect to } Q_{s y m}\end{array}$ \\
\hline$\preceq G L_{c}$ & $\begin{array}{l}\text { c-dominance relation } \\
\text { (conditional generalised Lorenz dominance relation) }\end{array}$ & $\begin{array}{l}\text { Extension of } \preceq_{G L} \text {, includes preference information. } \\
\text { Dominance with respect to } Q_{s y m}\left(\preceq_{R}\right)\end{array}$ \\
\hline$C\left(R ; z^{k}\right)$ & Cone generated by preference information $\preceq_{R}$ & \\
\hline Upper generators of $C\left(R ; z^{k}\right)$ & Alternatives $z^{i} \in R$ & \\
\hline Lower generator of $C\left(R ; z^{k}\right)$ & Alternative $z^{k}$ & \\
\hline$P\left(R ; z^{k}\right)$ & Polyhedron spanned by $z^{1}, \ldots, z^{k}$ (distributions in set $R \cup z^{k}$ ) & \\
\hline$L_{\text {asym }}$ or $L_{a s y m}\left(z^{k}\right)$ & $\begin{array}{l}\text { Given } \preceq_{R} \text { the set of points conditionally dominated } \\
\text { by } z^{k} \text { (asymmetric case) }\end{array}$ & $\left\{z: z \leq z^{\prime}\right.$ for some $\left.z^{\prime} \in C\left(R ; z^{k}\right)\right\}$ \\
\hline$U_{\text {asym }}$ or $U_{\text {asym }}\left(z^{k}\right)$ & $\begin{array}{l}\text { Given } \preceq_{R} \text { the set of points conditionally dominating } \\
z^{k} \text { (asymmetric case) }\end{array}$ & $\left\{z: z^{\prime} \leq z\right.$ for some $\left.z^{\prime} \in P\left(R ; z^{k}\right)\right\}$ \\
\hline$\Pi(R)$ & The set of all permutations of the distributions in set $R$ & \\
\hline Lower set $\left(L\right.$ or $\left.L\left(z^{k}\right)\right)$ & $\begin{array}{l}\text { Given } \preceq_{R} \text { the set of points c-dominated by } z^{k} \\
\text { (symmetric case) }\end{array}$ & $\begin{array}{l}\left\{z^{\prime}: z^{\prime} \preceq_{G L} z\right. \\
\text { for some } z \in C\left(\Pi\left(R \cup z^{k}\right) ; \Pi^{s}\left(z^{k}\right)\right) \\
\left.\text { for some permutation } \Pi^{s}\left(z^{k}\right) \text { of } z^{k}\right\} \text {. }\end{array}$ \\
\hline Upper set $\left(U\right.$ or $\left.U\left(z^{k}\right)\right)$ & $\begin{array}{l}\text { Given } \preceq_{R} \text { the set of points c-dominating } z^{k} \\
\text { (symmetric case) }\end{array}$ & $\begin{array}{l}\left\{z^{\prime}: z \preceq G L z^{\prime} \text { for some }\right. \\
\left.z \in P\left(\Pi\left(R \cup z^{k}\right) ; z^{k}\right)\right\}\end{array}$ \\
\hline$\hat{\Pi}\left(\overrightarrow{z^{k}}\right)$ & $\begin{array}{l}\text { The set of }(p-1) \text { distributions, each of which is obtained by } \\
\text { swapping two consecutive elements in } \overrightarrow{z^{k}}\end{array}$ & $\begin{array}{l}\hat{\Pi}\left(\overrightarrow{z^{k}}\right)=\left\{z^{\prime}: z_{i^{\prime}}^{\prime}={\overrightarrow{z^{k}}}_{i^{\prime}+1}, z_{i^{\prime}+1}^{\prime}={\overrightarrow{z^{k}}}_{i^{\prime}}\right. \\
\left.z_{i}^{\prime}=\overrightarrow{z_{i}^{k}} \forall i \neq i^{\prime}, i^{\prime}+1 \text { for some } i^{\prime} \in I\right\} .\end{array}$ \\
\hline
\end{tabular}

$U_{\text {asym }}\left(z^{k}\right)=\left\{z \mid z^{\prime} \leq z\right.$ for some $\left.z^{\prime} \in P\left(R ; z^{k}\right)\right\}$

Similar to Definition 3, we can define conditional dominance for the asymmetric settings.

Definition 4. For any $z^{1}, \quad z^{2} \in \mathbb{R}^{p}, z^{1} \preceq_{c} z^{2} \Leftrightarrow u\left(z^{1}\right) \leq u\left(z^{2}\right)$ $\forall u(.) \in Q\left(\preceq_{R}\right)$, where $Q\left(\preceq_{R}\right)$ is the set of social evaluation functions that are increasing, strictly quasiconcave and consistent with $\preceq_{R}$.

The following result can be used to check this conditional dominance in asymmetric settings:

Theorem 3 (Korhonen et al., 1984). ${ }^{3}$ Consider $z, z^{\prime} \in \mathbb{R}^{p}$. Then $z$ $\preceq_{c} z^{\prime}$ if the following hold:

(i) $z \in L_{\text {asym }}\left(z^{k}\right)$.

(ii) $z^{\prime} \in U_{\text {asym }}\left(z^{k}\right)$.

Example 4. To illustrate the application of the Theorem 3, consider the example in Fig. 3a and b. Consider the distribution defined by point $(2,6)$. Fig. 3a shows the region of distributions that dominate $(2,6)$ (etched region) and the set of points that are dominated by $(2,6)$ (dotted region) in the absence of preference information. Fig. $3 \mathrm{~b}$ shows the impact of introducing preference information, namely $(3,4)$ is preferred to $(2,6)$. Both the regions of points dominated by $(2,6)$ and the set of points that dominate $(2,6)$ increase as seen in the figure. These sets are again derived by using the convexity property of the indifference curves of the evaluation function as explained in the previous section. Any distribution $z$ falling within the dotted region is (conditionally) dominated by any distribution $z^{\prime}$ falling within the etched region. By use of Theorem 3, we can check (by solving systems of linear inequalities) whether this is the case for any arbitrary points $z$ and

\footnotetext{
${ }^{3}$ Although the original results do not impose the symmetry assumption they also hold for the case with symmetry due to the axiom of convexity, which is common to both cases.
}

$z^{\prime}$ and, if so, the original ranking of distributions can be refined by adding the information that $z \preceq_{c} z^{\prime}$.

Theorem 3 has been traditionally applied when there is no a priori assumption that the SP is indifferent between all permutations of a distribution (in the preceding example, we made no use of the symmetry assumption about the SP's preferences). If this were the case, however, the ranking could be refined further. To see this, consider the same example in a symmetric setting. Fig. $4 \mathrm{a}$ and $\mathrm{b}$ shows the dominating and dominated regions with and without preference information respectively. In Fig. 4a, $(2,6)$ is considered equally good as $(6,2)$, by symmetry, and so there are now two dominated regions. In Fig. $4 \mathrm{~b}$, symmetry dictates that any of $(3,4)$ or $(4,3)$ is preferred to any of $(2,6)$ and $(6,2)$ and so both dominating and dominated regions increase. For any two distributions $z$ and $z^{\prime}$ such that $z$ falls within any of the two (dotted) enlarged dominated regions and $z^{\prime}$ falls within the (etched) dominating region we can again infer that $z \preceq_{G L_{c}} z^{\prime}$.

In addition to the usefulness of preference information in a symmetric setting, the above example also illustrates the increase in computational complexity due to symmetry. As can be seen, we need to perform checks by taking into account every permutation of the distributions over which preferences are provided. With an increase in the number of entities and the reference distributions considered, this becomes prohibitive. The results that we introduce below alleviate this problem.

Let $I$ be the index set of entities, i.e. $I=\{1,2, \ldots, p\}$. For our results, we will use the following two sets of permutations of the reference distributions in $R$ and distribution $z^{k}$ :

$\Pi(R)=\left\{z^{\prime}: z^{\prime}=\Pi^{s}(z)\right.$ for some permutation $\Pi^{s}(z)$ of $\left.z \in R\right\}$.

$\hat{\Pi}\left(\overrightarrow{z^{k}}\right)=\left\{z^{\prime}: z_{i^{\prime}}^{\prime}={\overrightarrow{z^{k}}}_{i^{\prime}+1}, z_{i^{\prime}+1}^{\prime}={\overrightarrow{z^{k}}}_{i^{\prime}}, \quad z_{i}^{\prime}=\overrightarrow{z_{i}^{k}}\right.$

$\forall i \neq i^{\prime}, i^{\prime}+1$ for some $\left.i^{\prime} \in I\right\}$. 


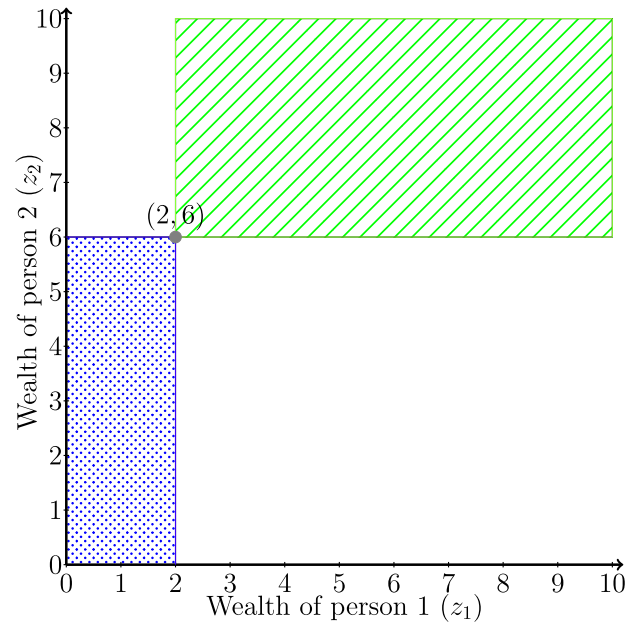

(a) No preference information in an asymmetric setting

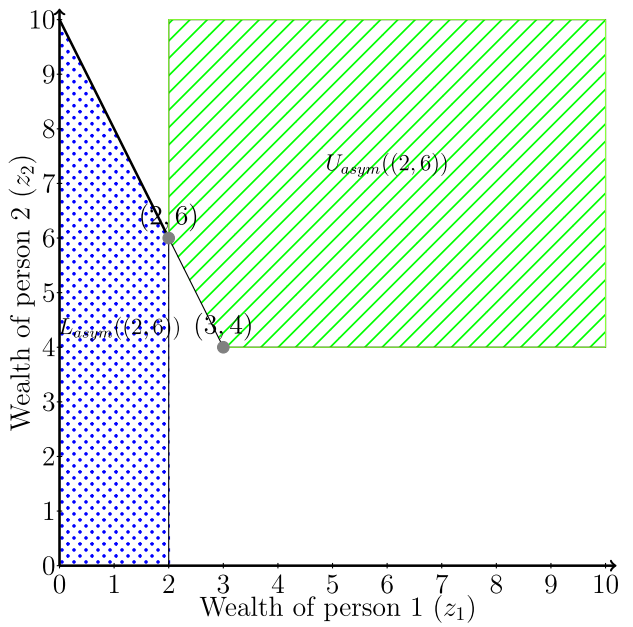

(b) Preference information in an asymmetric setting

Fig. 3. Asymmetric setting.

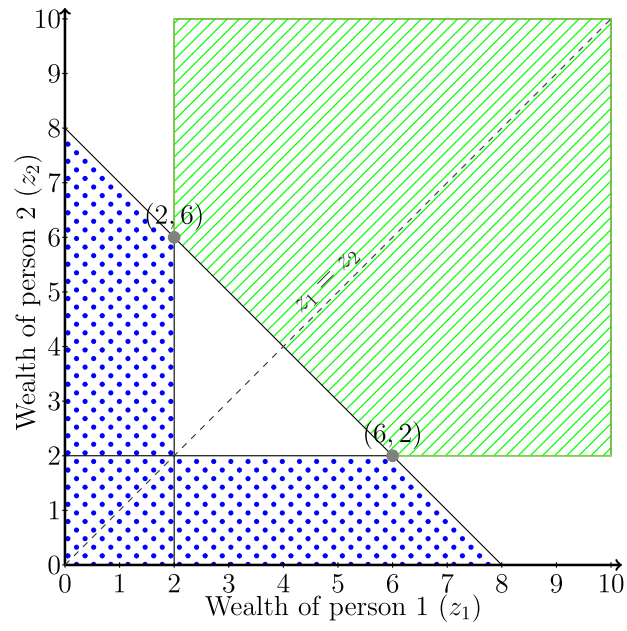

(a) Before preference information is elicited

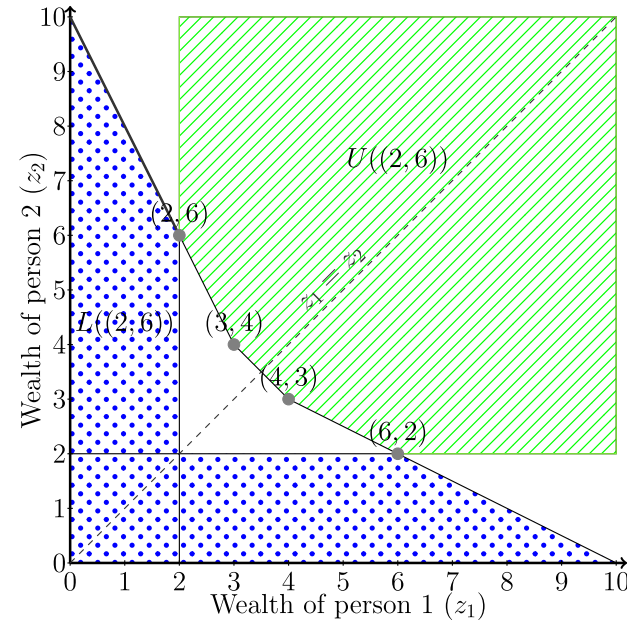

(b) After preference information is elicited

Fig. 4. Symmetric setting.

$\Pi(R)$ is the set of all possible permutations of the reference distributions and $\hat{\Pi}\left(\overrightarrow{z^{k}}\right)$ is the set of permutations of $\overrightarrow{z^{k}}$, each of which is obtained by swapping two consecutive elements of $\overrightarrow{z^{k}}$.

Using $\Pi(R)$, we may now formally express the lower and upper sets $L\left(z^{k}\right)$ and $U\left(z^{k}\right)$ through these cones and polyhedrons:

$$
\begin{aligned}
L\left(z^{k}\right)= & \left\{z \mid z \preceq_{G L} z^{\prime} \text { for some } z^{\prime} \in C\left(\Pi\left(R \cup z^{k}\right) ; \Pi^{s}\left(z^{k}\right)\right)\right. \\
& \text { for some permutation } \left.\Pi^{s}\left(z^{k}\right) \text { of } z^{k}\right\} . \\
U\left(z^{k}\right)= & \left\{z \mid z^{\prime} \preceq_{G L} z \text { for some } z^{\prime} \in P\left(\Pi\left(R \cup z^{k}\right) ; \Pi^{s}\left(z^{k}\right)\right)\right. \\
& \text { for some permutation } \left.\Pi^{s}\left(z^{k}\right) \text { of } z^{k}\right\} .
\end{aligned}
$$

Theorem 4. Consider $z, z^{\prime} \in \mathbb{R}^{p}$. Then $z \preceq_{G L c} z^{\prime}$ if the following hold:

(i) $z \in L\left(z^{k}\right)$.

(ii) $z^{\prime} \in U\left(z^{k}\right)$.

\section{Proof.}

(i) $z \in L\left(z^{k}\right)$ then $z \preceq_{G L} z^{\prime}$ for some $z^{\prime} \in C\left(\Pi\left(R \cup z^{k}\right)\right.$; $\left.\Pi^{s}\left(z^{k}\right)\right)$ for some permutation $\Pi^{s}\left(z^{k}\right)$ of $z^{k}$. This implies $z^{\prime} \preceq_{c} \Pi^{s}\left(z^{k}\right)$. Note that $\preceq_{c}$ implies $\preceq_{G L_{c}}$ (as the set of symmetric quasiconcave functions is a subset of the set of quasiconcave functions) hence $z^{\prime} \preceq_{G L_{c}} \Pi^{s}\left(z^{k}\right)$. $\preceq_{G L_{c}}$ is symmetric, therefore $z^{\prime} \preceq_{G L_{c}} z^{k}$. Then $z \preceq_{G L} z^{\prime} \preceq_{G L_{c}} z^{k}$, implying $z \preceq_{G L_{c}} z^{k}$.

(ii) $z^{\prime} \in U\left(z^{k}\right)$ then $z^{\prime \prime} \succeq_{G L} z^{\prime}$ for some $z^{\prime \prime} \in P\left(\Pi\left(R \cup z^{k}\right)\right.$; $\left.\Pi^{s}\left(z^{k}\right)\right)$ for some permutation $\Pi^{s}\left(z^{k}\right)$ of $z^{k}$ \}. This implies $\Pi^{s}\left(z^{k}\right) \leq_{c} z^{\prime \prime}$, hence $\Pi^{s}\left(z^{k}\right) \preceq_{G L_{c}} z^{\prime \prime} . \preceq_{G L_{c}}$ is symmetric, therefore $z^{k} \preceq_{G L_{c}} z^{\prime \prime}$. We have $z^{k} \preceq_{G L_{c}} z^{\prime \prime} \preceq_{G L} z^{\prime}$, implying $z^{k} \preceq_{G L_{c}} z^{\prime}$.

From parts (i) and (ii) $z \preceq_{G L_{c}} z^{k} \unlhd_{G L_{c}} z^{\prime}$, then $z \preceq_{G L_{c}} z^{\prime}$.

The sets $L\left(z^{k}\right)$ and $U\left(z^{k}\right)$ above contain distributions that are conditionally generalised Lorenz dominated by, or conditionally generalised Lorenz dominate $z^{k}$. It follows that any distribution in $L\left(z^{k}\right)$ is conditionally generalised Lorenz dominated by any distribution in $U\left(z^{k}\right)$. Therefore these sets provide a mechanism for checking conditional generalised Lorenz dominance between any pair of distributions, $z$ and $z^{\prime}$, specifically by checking membership of either in $L\left(z^{k}\right)$ or $U\left(z^{k}\right)$. This would involve solving two LPs for each permutation. As mentioned before, this can be computationally prohibitive, as it requires a membership check for every single 
one of the $p$ ! permutations $\Pi^{s}\left(z^{k}\right)$ of $z^{k}$ and in each check all permutations of the reference alternatives $z^{i} \in R$ should be considered. However, our result below shows that we can completely remove the need for considering all permutations of $z^{k}$ and $z^{i} \in R$.

In particular, instead of $L\left(z^{k}\right)$ and $U\left(z^{k}\right)$, we will use the following:

$$
\begin{aligned}
\hat{L}\left(z^{k}\right) & =\left\{z \mid z \preceq_{G L} z^{\prime} \text { for some } z^{\prime} \in C\left(\vec{R} \cup \hat{\Pi}\left(\overrightarrow{z^{k}}\right) ; \overrightarrow{z^{k}}\right)\right\} . \\
\hat{U}\left(z^{k}\right) & =\left\{z \mid z^{\prime} \preceq_{G L} z \text { for some } z^{\prime} \in P\left(\vec{R} ; \overrightarrow{z^{k}}\right)\right\} .
\end{aligned}
$$

Theorem 5. Consider two distributions $z, z^{\prime} \in \mathbb{R}^{p}$. The following are equivalent:

(i) $z \in L\left(z^{k}\right)$ and $z^{\prime} \in U\left(z^{k}\right)$

(ii) $z \in \hat{L}\left(z^{k}\right)$ and $z^{\prime} \in \hat{U}\left(z^{k}\right)$.

Corollary 1. Consider two distributions $z, z^{\prime} \in \mathbb{R}^{p}, z \in \hat{L}\left(z^{k}\right)$ and $z^{\prime} \in$ $\hat{U}\left(z^{k}\right)$ implies $z \preceq G L c z^{\prime}$.

To summarise, the result above allows for checking conditional generalised Lorenz dominance between a pair of distributions in an analogous way to Theorem 3 , but also taking symmetry into account, therefore further refining the ranking of distributions. At the same time, the computational burden of accounting for symmetry is avoided, as it suffices to work with the ordered vector $\overrightarrow{z^{k}}$ as the lower generator, instead of considering all permutations $\Pi^{s}\left(z^{k}\right)$ of $z^{k}$ separately. Moreover instead of using $\Pi(R)$ in the set of upper generators we only consider $\vec{R}$, i.e., the ordered vectors of distributions in reference set $R$. Checking dominance involves solving a LP per each membership check for $\hat{L}\left(z^{k}\right)$ and $\hat{U}\left(z^{k}\right)$. The details of this (and the proof of the Theorem) are given in the Appendix (Appendix A.1).

In addition to the above, further simplification and computational savings are possible for the special case where we are considering a single reference distribution $z^{i}$ at a time and use twopoint cones of the form $C\left(z^{i} ; z^{k}\right)$ (A two-point cone is a cone that consists of one upper generator and one lower generator). In particular, define:

$$
\begin{aligned}
L\left(z^{k}\right)= & \left\{z \mid z \preceq_{G L} z^{\prime} \text { for some } z^{\prime} \in C\left(\Pi^{r}\left(z^{i}\right) ; \Pi^{s}\left(z^{k}\right)\right)\right. \\
& \text { for some permutations } \Pi^{r}\left(z^{i}\right) \text { and } \Pi^{s}\left(z^{k}\right) \\
& \text { of } z^{i} \text { and } z^{k}, \text { for some } z^{i} \in R \text { or } z^{\prime} \\
& \in C\left(\Pi^{r}\left(z^{k}\right) ; \Pi^{s}\left(z^{k}\right)\right) \\
& \text { for some permutations } \\
& \left.\Pi^{r}\left(z^{k}\right) \text { and } \Pi^{s}\left(z^{k}\right) \text { of } z^{k}\right\} . \\
\bar{L}\left(z^{k}\right)= & \left\{z \mid z \preceq_{G L} z^{\prime} \text { for some } z^{\prime} \in C\left(\overrightarrow{z^{i}} ; \overrightarrow{z^{k}}\right)\right. \\
& \text { for some } \left.z^{i} \in R\right\}
\end{aligned}
$$

Theorem 6. Consider two distributions $z, z^{\prime} \in \mathbb{R}^{p}$. The following are equivalent:

$$
\begin{aligned}
& \text { (i) } z \in L\left(z^{k}\right) \text { and } z^{\prime} \in U\left(z^{k}\right) \\
& \text { (ii) } z \in \bar{L}\left(z^{k}\right) \text { and } z^{\prime} \in \hat{U}\left(z^{k}\right) \text {. }
\end{aligned}
$$

To summarise, by considering separately every $z^{i} \in R$, and using two-point cones, we may reduce the computational burden, as we can disregard taking permutations of $z^{k}$ into account and instead just use the ordered vector $\overrightarrow{z^{k}}$ (see Korhonen et al., 1984 for a discussion on the difference between using two-points cones and larger cones in settings without symmetry assumption. Their computational experiments indicate that using larger cones eliminates more alternatives than using two-point cones. On the other hand the LPs solved considering two-point cones are easier to handle as they are of smaller size, hence there is a trade-off between com- putational gain and information gain). The proof of the Theorem is provided in Appendix A.2.4

The mathematical models solved to check whether a distribution is in the $\hat{L}\left(z^{k}\right)$ (or $\bar{L}\left(z^{k}\right)$ ) or $\hat{U}\left(z^{k}\right)$ given preference information $\preceq_{R}$ are provided in Appendix B. In the next section we propose an interactive ranking algorithm which is based on our theoretical results.

\section{Interactive algorithm}

We propose an algorithm that can be used to obtain a ranking of a given discrete set of distributions. Suppose that we are given a finite number of distributions each showing a distribution profile for $p$ entities. We can summarise our algorithm with the following main steps:

S.1. Check whether any distribution is generalised Lorenz dominated by the other for each pair of distributions. This check is performed by the dominancecheck subroutine in Algorithm 1.

\section{Algorithm 1 Interactive algorithm. \\ Read problem data and initialise the parameters using Initialisa- tion subroutine \\ Check GL dominance between each pair of distributions using Dominancecheck subroutine \\ Repeat}

Get preference information from the SP using Getinfo subroutine

newinfo=1 //This parameter is used to check whether any new information is obtained that can allow us to generate new cones and polyhedra

Repeat

Perform the checks related to L and U using Conegeneration subroutine

Until newinfo $=0$

Count the number of distributions whose ranks are known using Countassigned subroutine

Until $n$-unassigned $=n$ or CPUtime $>1800 \quad / / n$ is the number of distributions

Display results and performance measure values

S.2. Select $k$ distributions $(k \geq 2)$ based on a predetermined rule. Get the preference information from the SP by asking her to compare these distributions. Denote the least preferred distribution as $z^{k}$ and the rest as $z^{i}$ for $i=1,2, \ldots, k-1$. This is performed by the getinfo subroutine in Algorithm 1.

S.3. Based on the preference information obtained, check for each distribution $z$ whether $z \in L\left(z^{k}\right)$. If not, then check whether $z^{k} \in U\left(z^{k}\right)$. If any new information is obtained, which would allow new cones and polyhedra to be generated, repeat this step. We perform these checks, respectively, by solving two linear programming models, $L P_{1}$ and $L P_{2}$, discussed in Appendix B. Conegeneration subroutine in Algorithm 1 performs these operations.

S.4. Update the results accordingly (in the Countassigned subroutine). If the result is not satisfactory according to some predetermined stopping criterion, continue with Step 2.

The pseudocode of the algorithm is as follows.

See Appendix $C$ for detailed explanation of the subroutines.

\footnotetext{
${ }^{4}$ Note that Theorem 6 applies to situations where $p>2$ as long as only two-point cones and polyhedra are used. However, it is not generalisable to cases where we use larger cones. This is because for two distributions $z^{i}$ and $z^{k}$ such that $z^{i} \succ z^{k}$, we can claim for a distribution $z$ that, if there is a $z^{\prime \prime} \in C\left(z^{i} ; z^{k}\right): z \preceq_{G L} z^{\prime \prime}$ then there is $z^{\prime \prime \prime} \in C\left(\overrightarrow{z^{i}}, \overrightarrow{z^{k}}\right): z \preceq_{G L} z^{\prime \prime \prime}$. However, for any $k$ vectors $z^{1}, \ldots, z^{k} \in \mathbb{R}^{p}$ such that $z^{i} \succ z^{k}$ for all $i \neq k$ and $z \in \mathbb{R}^{p}$ we cannot claim for a distribution $z$ that, if there is a $z^{\prime \prime} \in C(R$; $\left.z^{k}\right): z \preceq_{G L} z^{\prime \prime}$ then there is a $z^{\prime \prime \prime} \in C\left(\vec{R} ; \overrightarrow{z^{k}}\right): z \preceq_{G L} z^{\prime \prime \prime}$. See the counterexample in Appendix A.2.
} 
Table 4

\begin{tabular}{|c|c|c|c|c|c|c|c|}
\hline Voucher & 1 & 2 & 3 & Voucher & 1 & 2 & 3 \\
\hline 1 & 90 & 90 & 150 & 6 & 50 & 75 & 230 \\
\hline 2 & 75 & 125 & 125 & 7 & 65 & 125 & 150 \\
\hline 3 & 85 & 85 & 180 & 8 & 80 & 115 & 115 \\
\hline 4 & 95 & 95 & 110 & 9 & 65 & 130 & 135 \\
\hline 5 & 70 & 105 & 160 & 10 & 85 & 95 & 155 \\
\hline
\end{tabular}

\section{Results}

\subsection{Tests with real subjects}

The proposed interactive decision support system was tested with fourteen individuals with a variety of backgrounds in economics, mathematics, statistics and engineering to see whether it is usable. These individuals were selected as thoughtful people with quantitative backgrounds. We presented the subjects the following cover story, which involved ranking 10 distributions (options) each of which represents an allocation of a good over three indistinguishable people.

You are giving three presents to three indistinguishable nephews or nieces (they are triplets so there are no age differences) for their 21st birthday. One of them likes books; one likes $C D$ s and the other likes clothes, so you want to give them vouchers for these items. You want to spend 1270 altogether, but some shops will give you vouchers with a total value $>£ 270$ for this money. For example, you can buy vouchers at shop A which will give you a voucher for each niece for $£ 90$ (hence with a total value of $£ 270$, equitably distributed) or at shop B which will give you $2 £ 80$ vouchers and a £130 voucher (with a total value of $£ 290$ but less equitably distributed). Which do you prefer? Suppose that you have a list of alternative vouchers from different shops (10 options in total) and you want to rank these from best to the worst.

The option set is given in Table 4. Note that only one of the distributions in the option set is generalised Lorenz dominated.

Our tests involved the use of two procedures. The first procedure (Procedure 1) was based on asking holistic comparisons between pairs of options. The second procedure (Procedure 2) was based on the use of an additive power evaluation (social welfare function (SWF)) of the form $\left(\sum_{i=1}^{p}\left(z_{i}\right)^{\alpha}\right)^{\frac{1}{\alpha}}$. The power SWF of procedure 2 was parameterised, i.e. a value for $\alpha$ was found, by asking a single indifference question to the subject. The indifference question was based on finding the equally distributed equivalent (EDE) of a given option. Specifically, the subject was given $(90,90,150)$ and asked to provide a value $x$ such that s/he valued $(90,90,150)$ and $(x, x, x)$ the same (i.e. $\mathrm{s} /$ he was indifferent). After finding the corresponding $\alpha$ value, we obtained a full ranking.

With each subject, we used both procedures to obtain two (potentially different) rankings of the options. At the end, we asked the subjects the following questions:

1. How easy did you find it to make holistic comparisons between options (relative to finding an EDE)? (very easy, quite easy, neither easy nor difficult, quite difficult, very difficult).

2. How satisfactory do you find the interactively derived ranking versus the SWF derived ranking? (more satisfactory, quite satisfactory, neither satisfactory nor unsatisfactory, quite unsatisfactory, very unsatisfactory).

We note here that our aim in performing these tests is in the spirit of an existence proof: we want to establish whether people could use the procedure, not to establish the definitive superiority of Procedure 1 vs. Procedure 2 (as we would expect that different people would prefer different questioning modes). In any case, such a comparison would not be appropriate as our experimental
Table 5

Results of the tests with real subjects.

\begin{tabular}{llllll}
\hline Subject & $\begin{array}{l}\text { Solution } \\
\text { time }\end{array}$ & $\begin{array}{l}\text { Number of } \\
\text { questions }\end{array}$ & Subject & $\begin{array}{l}\text { Solution } \\
\text { time }\end{array}$ & $\begin{array}{l}\text { Number of } \\
\text { questions }\end{array}$ \\
\hline A & 1234 & 20 & H & 898 & 19 \\
B & 727 & 13 & I & 765 & 19 \\
C & 842 & 15 & J & 753 & 17 \\
D & 810 & 14 & K & 819 & 18 \\
E & 1087 & 19 & L & 616 & 16 \\
F & 873 & 20 & M & 810 & 18 \\
G & 600 & 15 & N & 692 & 18 \\
\hline
\end{tabular}

design was not counterbalanced to guard against order effects, as one of the subjects noted.

One of the main observations we made in our experiments is that the problem we try to handle is cognitively challenging due to the tradeoff between efficiency and equity. This justifies the importance of designing appropriate decision support which relies on inputs collected from the SP in a way that is natural to him/her (like our holistic comparisons) and which provides satisfactory results.

Table 5 summarises some information on the experiments in terms of the solution time of Procedure 1 (in seconds) and the number of questions answered. As seen in the table the whole procedure took less than 20 minutes for most trials and the number of questions answered was at most 20.

All of the subjects provided a positive feedback in terms of the usability of our method. We also asked about acceptability of the distribution question (Question 1) but without getting consensus about whether Procedure 1 or Procedure 2 was preferred (seven subjects found making holistic comparisons quite difficult while six subjects found it quite easy, and one subject found it very easy relative to finding an EDE). In terms of the satisfaction derived from the two rankings (Question 2), the feedback was also mixed. One subject found Procedure 1 neither satisfactory nor unsatisfactory, four found it quite satisfactory, seven found it more satisfactory and two found it quite unsatisfactory relative to Procedure 2.

Overall, our small set of trials indicated that the procedure is usable and is competitive with an EDE-based approach, Procedure 2. We also note that our method does not make the strong structural assumptions which are required by Procedure 2, in the sense that no parametric form is assumed.

\subsection{Computational experiments}

Our initial motivation for this study was to provide a ranking procedure for comparing income distributions. In order to test our procedure in this setting, we used income distribution information of different countries from the World Bank (WB, 2011) and UNU-WIDER (United Nations University-World Institute for Development Economics Research) (WIDER, 2011) databases. We used the quintile values to represent a country's income distribution. We performed tests with smaller discrete datasets (for $n$ values of $14,15,26,39,54$ and 66) but we found that many relations in these datasets are already determined by generalised Lorenz dominance and so these datasets do not allow our procedure to demonstrate its full potential.

To demonstrate the full potential of the procedure, we explore its performance in an environment where none or only some of the distributions are generalised Lorenz dominated. Note that this is the sort of dataset which might be generated by one of the previously discussed algorithms (e.g. by Baatar \& Wiecek, 2006; Kostreva \& Ogryczak, 1999; Kostreva et al., 2004; Ogryczak, 2000; Ogryczak et al., 2008) for exhaustively generating or sampling the efficient set in the context of some optimisation problem such as 
might naturally occur in, for example, a telecommunication or logistics application.

To test the performance of our procedure for such settings, distributions are generated randomly from a uniform distribution using MATLAB's random number generator. In this set only nondominated distributions are generated (a distribution is nondominated if there is no other distribution in the set that generalised Lorenz dominates it).

We simulated the SP's responses using an underlying evaluation function. Three types of underlying evaluation functions are used in the experiments:

1. Linear evaluation function

$$
U\left(z^{i}\right)=\sum_{j=1}^{p} \overleftarrow{w}_{j} \vec{z}_{j}^{i}
$$

where $w_{j}, j=1, \ldots, p$ are generated from a uniform distribution between 0 and 1 .

2. Product function

$$
U\left(z^{i}\right)=\prod_{j=1}^{p} z_{j}^{i}
$$

\section{Tchebycheff evaluation function}

$U\left(z^{i}\right)=\vec{z}_{1}^{i}$

We use the following performance measures used to evaluate performance of approach and the algorithm.

1. CPU time in seconds

2. Number of $L P_{1}$ problems solved

3. Number of $L P_{2}$ problems solved

4. Number of binary comparisons gathered from the SP

5. Ratio of the binary comparisons gained through the cdominance approach

The definitions of measures 1,2, 3 and 4 are clear. Let us explain measure 5 .

In order to achieve a complete ranking of $n$ distributions, one has to know the relation between each pair of distributions in this set. Hence, we should know the result of $\left(\begin{array}{l}n \\ 2\end{array}\right)$ binary comparisons. At each iteration one binary comparison is asked to the SP, hence, in $t$ iterations $t$ such comparisons will be provided. We then calculate the ratio of the binary comparisons gathered from the SP, which we call qratio as follows:

qratio $=t /\left(\begin{array}{l}n \\ 2\end{array}\right)$

In the Dominancecheck subroutine of the algorithm we find the number of pairwise generalised Lorenz dominance relations. Let us denote it by $d$. We also find the ratio of the generalised Lorenz dominance relations which is a property of the problem set rather than a performance measure. We call it dratio and calculate as follows:

dratio $=d /\left(\begin{array}{l}n \\ 2\end{array}\right)$

Ratio of the binary comparisons gained through the cdominance approach (gainratio): Similar to qratio, this measure is used to see the amount of information that we gain by using the c-dominance approach. It is calculated as follows:

gainratio $=1-$ qratio - dratio

The optimal solutions of the LPs are found by using CPLEX 12.2. We set a termination limit of 30 minutes to the algorithm. All experimentations are done in Intel Core i5 2.27 gigahertz, 4 gigabyte RAM. The algorithm is coded with MATLAB.
We now discuss the performance of our algorithm. For each combination of the settings discussed above, we generate instances starting with $n=10$ and $p=2$, increasing them in increments of 10 and 1, respectively. For each combination of evaluation function, $p$ and $n$ values, 10 problem instances are generated. For the linear evaluation function case, objective function weights are randomly generated for each problem instance and then ordered in the nondecreasing manner. The average performance measure values over the 10 instances are shown in Table 6. Recall that in each problem instance none of the distributions are generalised Lorenz dominating each other, i.e., dratio $=0$. Hence this set consists of more difficult problems in that sense.

We can find complete rankings for problems with up to 70 distributions when the number of parties, $p$, is two, and up to 40 distributions when $p$ is three, four and five in our time limit of 30 minutes.

These results reveal the contribution of using c-dominance (conditional generalised Lorenz dominance). The minimum average gainratio value is 0.49 , that is, at least about $50 \%$ of the binary comparisons are provided by the lower and upper sets ( $L$ and $U$ ). This indicates a satisfactory performance for the approach. Note here that in some instances gainratio is seen as 1 , which is due to rounding.

We can see the effect of problem size on the performance of the algorithm and on the amount of information gained.

As can be observed from the table when the number of distributions, $n$, increases gainratio increases. Hence for constant $p$, the contribution of the conditional dominance approach to the solution increases as $n$ increases. Note that with increasing $n$, the number of questions increases, resulting in an increase in the number of cones/polyhedra generated. Moreover as $n$ increases so does the the number of LPs solved per $L$ and $U$. As a result, we observe an increase in the number of LPs solved and the solution time.

The effect of number of entities, $p$, is also notable in the performance of the approach. As $p$ increases the number and ratio of the comparisons required from the SP increase. As a result of the increase in the ratio of the comparisons required, the ratio of information gained decreases. Moreover the increase in the number of comparisons provided by the SP leads to an increase in the number of LP models solved and in turn an increase in solution time.

It is also observed that the effects of $p$ and $n$ are consistent over the three types of evaluation functions used.

\section{Discussion}

In this study we consider a method to incorporate preference information for equitability problems. We are motivated by the fact that problems involving equity concerns are widely encountered in real life, especially in the public sector. Such problems include, facility location, income distribution and resource/service allocation problems.

We extend the generalised Lorenz dominance concept by introducing the concept of conditional generalised Lorenz dominance (c-dominance): that is, generalised Lorenz dominance consistent with given preference information. We propose a method that, based on preference information about a distribution, characterises the lower set (the set of points c-dominated by that distribution) and the upper set (the set of points c-dominating that distribution) in a compact way. Characterising these sets in a compact way is not straightforward as symmetry brings combinatorial complexity to the problem. We provide theoretical results that help us handle this complexity and propose a tractable computational scheme for checking whether a given point belongs to one of these sets. In that sense, we extend the available methods in the multicriteria decision making (MCDM) literature such that they can be used for 
Table 6

Results of the computational experiments.

\begin{tabular}{|c|c|c|c|c|c|c|c|}
\hline $\begin{array}{l}\text { Evaluation } \\
\text { unction }\end{array}$ & $p$ & $n$ & Number of $L P_{1}$ & Number of $L P_{2}$ & $\begin{array}{l}\text { Solution time } \\
\text { (CPU seconds) }\end{array}$ & Gainratio & $\begin{array}{l}\text { Number of } \\
\text { questions asked }\end{array}$ \\
\hline \multirow[t]{20}{*}{1} & \multirow[t]{7}{*}{2} & 10 & 32.60 & 18.80 & 3.32 & 0.95 & 2.40 \\
\hline & & 20 & 192.40 & 149.30 & 21.66 & 0.96 & 7.90 \\
\hline & & 30 & 589.70 & 506.20 & 85.97 & 0.97 & 11.40 \\
\hline & & 40 & 944.20 & 813.00 & 121.55 & 0.98 & 13.70 \\
\hline & & 50 & 1714.20 & 1543.40 & 248.86 & 0.98 & 19.80 \\
\hline & & 60 & 2258.50 & 2005.80 & 341.17 & 0.99 & 17.80 \\
\hline & & 70 & 2159.40 & 1847.70 & 313.64 & 1.00 & 10.60 \\
\hline & \multirow[t]{5}{*}{3} & 10 & 100.90 & 94.10 & 12.27 & 0.68 & 14.50 \\
\hline & & 20 & 707.80 & 694.40 & 113.45 & 0.77 & 44.60 \\
\hline & & 30 & 2061.00 & 2011.50 & 320.35 & 0.84 & 70.60 \\
\hline & & 40 & 3446.10 & 3351.40 & 612.62 & 0.89 & 88.60 \\
\hline & & 50 & 6397.10 & 6308.70 & 1438.71 & 0.91 & 111.90 \\
\hline & \multirow[t]{4}{*}{4} & 10 & 129.50 & 128.70 & 16.43 & 0.54 & 20.90 \\
\hline & & 20 & 884.80 & 874.10 & 124.20 & 0.73 & 52.00 \\
\hline & & 30 & 2778.30 & 2757.50 & 472.12 & 0.78 & 95.80 \\
\hline & & 40 & 5737.00 & 5706.60 & 1278.61 & 0.82 & 140.90 \\
\hline & \multirow[t]{4}{*}{5} & 10 & 133.40 & 132.70 & 16.95 & 0.49 & 23.00 \\
\hline & & 20 & 1070.80 & 1065.30 & 149.77 & 0.64 & 67.70 \\
\hline & & 30 & 3100.40 & 3089.80 & 533.22 & 0.75 & 109.20 \\
\hline & & 40 & 6895.75 & 6881.38 & 1483.24 & 0.81 & 145.88 \\
\hline \multirow[t]{18}{*}{2} & \multirow[t]{7}{*}{2} & 10 & 46.70 & 33.40 & 5.07 & 0.91 & 4.10 \\
\hline & & 20 & 260.80 & 220.70 & 31.30 & 0.95 & 9.90 \\
\hline & & 30 & 739.70 & 669.60 & 96.78 & 0.96 & 17.50 \\
\hline & & 40 & 1399.70 & 1296.20 & 197.30 & 0.97 & 22.00 \\
\hline & & 50 & 2617.40 & 2466.60 & 418.67 & 0.98 & 28.10 \\
\hline & & 60 & 4026.70 & 3826.20 & 733.58 & 0.98 & 34.40 \\
\hline & & 70 & 5528.70 & 5267.70 & 1110.43 & 0.98 & 38.50 \\
\hline & \multirow[t]{4}{*}{3} & 10 & 98.50 & 92.90 & 12.11 & 0.64 & 16.20 \\
\hline & & 20 & 752.30 & 729.20 & 101.44 & 0.77 & 43.00 \\
\hline & & 30 & 2075.00 & 2035.50 & 325.55 & 0.81 & 82.20 \\
\hline & & 40 & 4332.30 & 4272.80 & 843.19 & 0.85 & 119.60 \\
\hline & \multirow[t]{4}{*}{4} & 10 & 123.50 & 122.40 & 15.80 & 0.52 & 21.70 \\
\hline & & 20 & 971.80 & 967.10 & 137.55 & 0.68 & 60.40 \\
\hline & & 30 & 2946.70 & 2934.10 & 508.64 & 0.76 & 104.70 \\
\hline & & 40 & 6290.67 & 6265.67 & 1213.64 & 0.80 & 152.67 \\
\hline & \multirow[t]{3}{*}{5} & 10 & 127.20 & 126.40 & 16.37 & 0.50 & 22.50 \\
\hline & & 20 & 1092.20 & 1088.70 & 153.25 & 0.66 & 64.70 \\
\hline & & 30 & 3380.00 & 3375.30 & 611.02 & 0.73 & 119.20 \\
\hline \multirow[t]{19}{*}{3} & \multirow[t]{7}{*}{2} & 10 & 27.20 & 11.80 & 2.51 & 0.96 & 1.80 \\
\hline & & 20 & 106.30 & 58.70 & 10.48 & 0.98 & 4.50 \\
\hline & & 30 & 263.00 & 157.40 & 26.79 & 0.99 & 3.20 \\
\hline & & 40 & 467.30 & 320.60 & 51.32 & 1.00 & 3.50 \\
\hline & & 50 & 573.20 & 375.60 & 73.15 & 1.00 & 3.40 \\
\hline & & 60 & 943.60 & 692.20 & 112.40 & 1.00 & 3.10 \\
\hline & & 70 & 1286.00 & 966.10 & 159.81 & 1.00 & 3.10 \\
\hline & \multirow[t]{4}{*}{3} & 10 & 96.00 & 90.30 & 11.77 & 0.67 & 14.90 \\
\hline & & 20 & 717.80 & 696.90 & 95.15 & 0.77 & 43.60 \\
\hline & & 30 & 2063.30 & 2019.90 & 323.04 & 0.84 & 71.70 \\
\hline & & 40 & 4189.10 & 4118.70 & 795.93 & 0.87 & 103.30 \\
\hline & \multirow[t]{4}{*}{4} & 10 & 128.20 & 127.00 & 16.56 & 0.49 & 23.10 \\
\hline & & 20 & 985.20 & 978.90 & 142.83 & 0.70 & 56.30 \\
\hline & & 30 & 2758.30 & 2740.40 & 562.76 & 0.78 & 95.10 \\
\hline & & 40 & 6040.00 & 6009.90 & 1324.71 & 0.81 & 147.10 \\
\hline & \multirow[t]{4}{*}{5} & 10 & 128.30 & 127.60 & 62.92 & 0.50 & 22.70 \\
\hline & & 20 & 1055.70 & 1052.00 & 531.56 & 0.66 & 64.20 \\
\hline & & 30 & 3275.90 & 3269.90 & 586.79 & 0.74 & 113.30 \\
\hline & & 40 & 4606.70 & 4594.50 & 1832.07 & 0.94 & 47.70 \\
\hline
\end{tabular}

problems with equity concerns without significantly increasing the computational efforts.

Our method does not rely on the assumption of an additive evaluation function and can handle non-additive preference models unlike stochastic dominance based approaches. It would in principle be possible to build a theory of conditional dominance in an environment where non-additive social evaluation functions are allowed using the machinery of Argyris, Morton, and Figueira (2014). But this method cannot capture symmetry and it is not obvious and it is beyond the scope of this paper how one should do it.
We check the performance of the suggested approach by using it in a ranking problem. Our tests with individuals indicate that people could use the procedure in real life settings. Our simulation results also suggest that the approach is computationally feasible for small to medium size problem settings. It is observed that the satisfactory performance of the algorithm is mostly due to the high ratio of information (at least $50 \%$ ) gained by the conditional generalised Lorenz dominance approach.

To the best of our knowledge, this study is the first extensive study that attempts to incorporate SP's preference information in equitability problems where the evaluation function is not as- 
sumed to be additive. This is also the first discussion on the convex cones approach in a symmetric environment and the first study that reports results for a ranking algorithm that uses the information from cones and polyhedra (conditional dominance approach) in this context.

This study can be extended by working more on the use of preference information in this context.

Our results in Theorem 4 of Section 4 provide only sufficient conditions for verifying conditional dominance in the symmetric setting. We note that this is also the case for the existing results in the literature for the asymmetric setting (see Theorem 3 of Section 4). Establishing whether our conditions are also necessary and if not, providing an extended set of conditions that would be both necessary and sufficient would settle the question as to whether further inferences about conditional dominance can be made and how this may be achieved. Clearly this is a promising extension to the methods introduced in this paper. Other than this, generalising the approach includes four main areas: Searching for alternative ways to handle computational complexity due to symmetry; using the approach in "selecting the best" and "sorting" problematiques (Roy, 1971), also in optimisation with different feasible sets; and performing an experimental study to use the approach as efficiently as possible. These potential research topics are discussed below.

1. Handling the computational complexity: Recall that the method we propose to handle computational complexities due to symmetry reduces many of the permutational computations. However, in $L P_{1}$ we still have to find some of the permutations of the lower generator of each cone. In the near future, more theoretical studies may be performed to see whether there exists a way to obtain the information without any permutational calculations or even if it is not possible, to design efficient algorithms for the solution of the resulting models.

2. Interactive algorithms for selecting the best and sorting problematiques: We have demonstrated the computational performance of the proposed approach in a ranking setting. The method can also be tested for selecting the best and sorting problematiques. Extending the method for sorting settings would especially be interesting since we use different ways to gather and use SP's preference information in different problematiques. For example, in a sorting environment, instead of taking pairwise comparisons or rankings, we may request him/her to assign the distributions into the classes.

3. Use of the method for optimisation problems with different feasible sets: One can study different problem environments where the feasible region is defined by constraints. Some examples are transportation problems, telecommunication network problems or location problems, where providing equitable service to the users of the system is an important concern. More research can be done to generalise the use of conditional dominance approach in such environments.

4. Experimental study on convex cones: While designing an algorithm the analyst makes various decisions regarding the ways to collect preference information from the SP. The performance of the algorithm may vary based on the size of the sample used for gathering preference information, the selection rule applied to select the distributions in the sample and the form of the information the SP provides. For example, given a set of $k$ distributions, we may require the SP to rank them or select the best/worst distributions in the sample. An interesting direction for further research would be to perform an experimental study to see the impact on computational performance and user behaviour of such decisions.

\section{Acknowledgements}

The authors are grateful to the subjects who participated in the tests. N. Argyris and Ö. Karsu are grateful for Newton-Katip Çelebi Researcher Links Travel Grant, which helped the preparation of the final revision of the manuscript.

\section{Appendix A. Proofs and models}

\section{A.1. Proof of Theorem 5}

We prove Theorem 5 in two parts by proving Theorems 7 and 8 below.

\section{A.1.1. Proof of Theorem 7}

Recall the definition of $U$ given preference information $\preceq_{R}$ : $U\left(z^{k}\right)=\left\{z \mid z^{\prime} \preceq_{G L} z\right.$ for some $z^{\prime} \in P\left(\Pi\left(R \cup z^{k}\right) ; \Pi^{s}\left(z^{k}\right)\right)$ for some permutation $\Pi^{s}\left(z^{k}\right)$ of $z^{k}$. That is, it is the set of points that generalised Lorenz dominate at least one point in the polyhedron generated by all the permutations of the distributions mentioned in $\preceq_{R}$ (distributions in set $\left.R \cup z^{k}\right)$. We show in Theorem 7 that $U\left(z^{k}\right)$ can be characterised compactly using the ordered vectors only. It shows that one can check whether a given distribution is in set $U$ without any permutational calculations.

Theorem 7. If $z \in U\left(z^{k}\right)$ then there is $z^{\prime} \in P\left(\vec{R} ; \overrightarrow{z^{k}}\right): z^{\prime} \preceq_{G L} z$ (hence $z \in \hat{U}\left(z^{k}\right)$, where $\hat{U}\left(z^{k}\right)=\left\{z \mid z^{\prime} \preceq_{G L} z\right.$ for some $\left.\left.z^{\prime} \in P\left(\vec{R} ; \overrightarrow{z^{k}}\right)\right\}\right)$.

We first prove Lemmas 1 and 2, which are auxiliary results that are used in multiple places in our proofs. We then prove Lemmas 3 and 4, which will be used in our main proof of Theorem 7. A short sketch of the proof strategy is as follows:

1. We first show in Lemma 1 that if one distribution is generalised Lorenz dominating another then it is obtainable from the latter by a finite-number of equity enhancing (PD) transfers. Using this result, we then provide an auxiliary result in Lemma 2 to be used in the proof of Lemma 3.

2. We show in Lemma 3 that for $z^{2}, z^{1} \in \mathbb{R}^{p}$, if $z \in P\left(z^{1} ; \overrightarrow{z^{2}}\right)$ then $\exists z^{\prime} \in P\left(\overrightarrow{z^{1}} ; \overrightarrow{z^{2}}\right): z^{\prime} \preceq_{G L} z$. This shows that using the ordered version of the upper generator is sufficient in terms of the information that can be obtained using a two-point polyhedron when the lower generator is ordered $\left(\overrightarrow{z^{2}}\right)$.

3. We then provide a more general result in Proposition 1 and show that for any $z^{2}, z^{1} \in \mathbb{R}^{p}$, if $z \in P\left(z^{1} ; z^{2}\right)$ then $\exists z^{\prime} \in$ $P\left(\overrightarrow{z^{1}} ; \overrightarrow{z^{2}}\right): z^{\prime} \preceq_{G L} z$. This shows that using the ordered versions of the upper and lower generators is sufficient in terms of the information that can be obtained using any two-point polyhedron.

4. We link the above results on the two-point polyhedra to the more general case of larger polyhedra of size $k$, by showing in Lemma 4 that, given $z^{i}$ such that $z^{i} \succ z^{k}, \forall z^{i} \in R$ we have the following:

If $z \in P\left(R ; z^{k}\right)$ then there exists $\lambda_{i}$ and $y^{i} \in P\left(z^{i} ; z^{k}\right)$ for $i=$ $1, \ldots, k-1$ such that $z=\sum_{i=1}^{k-1} \lambda_{i} y^{i}$ and $\sum_{i=1}^{k-1} \lambda_{i}=1$.

5. Finally, we prove Theorem 7 . We make use of the fact that each point $z$ in a $k$-point polyhedron (that is, $z \in P\left(R ; z^{k}\right)$ ) can be written as a convex combination of other points $y^{i}$ that are in the two point polyhedrons (generated by one of the upper generators and the lower generator of the $k$-point polyhedron) (see part 4 above). Each of these points $\left(y^{i}\right)$ generalised Lorenz dominate a point in $P\left(\overrightarrow{z^{i}} ; \overrightarrow{z^{k}}\right)$ for some $i$ (hence a point in $P\left(\vec{R} ; \overrightarrow{z^{k}}\right)$ as this polyhedron includes the smaller one). As set $\left\{z \mid z^{\prime} \preceq_{G L} z\right.$ for some $\left.z^{\prime} \in P\left(\vec{R} ; \overrightarrow{z^{k}}\right)\right\}$ is a convex set, any con- 
vex combination of these $y^{i} \mathrm{~s}$ is also in this set, hence there is $z^{\prime} \in P\left(\vec{R} ; \overrightarrow{z^{k}}\right): z^{\prime} \preceq_{G L} \sum_{i=1}^{k-1} \lambda_{i} y^{i}=z$.

We now give the detailed results. In a distribution, we call a transfer that takes an amount of good from a party and gives it to a poorer party without changing their relative positions to each other a Pigou-Dalton $(P-D)$ transfer.

Lemma 1. If $z \preceq_{G L} z^{\prime}$ then there is a $z^{\prime \prime} \in \mathbb{R}^{p}$ such that $z^{\prime \prime} \leq z^{\prime}$, and $z^{\prime \prime}$ is obtainable from $z$ by a finite number of $P-D$ transfers.

Proof of Lemma 1. See Ok (1998), Lemma 1 for the proof

Lemma 2. Let $z$ and $z^{\prime} \in \mathbb{R}^{p}$ such that $z_{i}=z_{i}^{\prime} \forall i \neq h, h+1$. Then $z$ $\preceq_{G L} z^{\prime}$ if and only if $\operatorname{Min}\left\{z_{h}, z_{h+1}\right\} \leq \operatorname{Min}\left\{z_{h}^{\prime}, z_{h+1}^{\prime}\right\}$ and $z_{h}+z_{h+1} \leq$ $z_{h}^{\prime}+z_{h+1}^{\prime}$.

\section{Proof of Lemma 2. Necessity:}

This proof comes from the definition of the generalised Lorenz dominance. $\operatorname{Min}\left\{z_{h}, z_{h+1}\right\} \leq \operatorname{Min}\left\{z_{h}^{\prime}, z_{h+1}^{\prime}\right\}$ and $z_{h}+z_{h+1} \leq z_{h}^{\prime}+z_{h+1}^{\prime}$ imply $\sum_{j=1}^{i} \overrightarrow{z_{j}} \leq \sum_{j=1}^{i} \overrightarrow{z_{j}^{\prime}} \forall i$, hence $z \preceq_{G L} z^{\prime}$.

\section{Sufficiency:}

From Lemma 1 , if $z \preceq_{G L} z^{\prime}$ then there is a $z^{\prime \prime} \in \mathbb{R}^{p}$ such that $z^{\prime \prime} \leq z^{\prime}$, and $z^{\prime \prime}$ obtainable from $z$ by a finite number of P-D transfers. Suppose that we have obtained a $z^{\prime \prime}$ such that $z^{\prime \prime} \leq z^{\prime}$ holds. Without loss of generality suppose that $\operatorname{Min}\left\{z_{h}, z_{h+1}\right\}=z_{h}$ and $\operatorname{Min}\left\{z_{h}^{\prime}, z_{h+1}^{\prime}\right\}=z_{h}^{\prime}$. If this is not the case, we can arrange them accordingly since we have symmetry.

Suppose that at least one of the following holds:

$z_{h}>z_{h}^{\prime}$ or $z_{h}+z_{h+1}>z_{h}^{\prime}+z_{h+1}^{\prime}$

$z_{i}^{\prime}=z_{i} \forall i \neq h, h+1$, so for $z^{\prime \prime} \leq z^{\prime}$ to hold, the P-D type transfer in distribution $z$ to obtain $z^{\prime \prime}$ should be from $z_{h+1}$ to $z_{h}$.

That is, $z_{h}^{\prime \prime}=z_{h}+\epsilon, z_{h+1}^{\prime \prime}=z_{h+1}-\epsilon$, where $0 \leq \epsilon \leq z_{h+1}-z_{h}$

$z_{i}^{\prime}=z_{i}=z_{i}^{\prime \prime} \forall i \neq h, h+1$ and $z^{\prime \prime} \leq z^{\prime} \Rightarrow z_{h}^{\prime} \geq z_{h}^{\prime \prime}=z_{h}+\epsilon$ and $z_{h+1}^{\prime} \geq z_{h+1}^{\prime \prime}=z_{h+1}-\epsilon$.

That is, $z_{h}^{\prime} \geq z_{h}$ and $z_{h}^{\prime}+z_{h+1}^{\prime} \geq z_{h}+z_{h+1}$, which is a contradiction to our initial assumption $\mathrm{A} 1$.

Lemma 3. For $z^{2}, z^{1} \in \mathbb{R}^{p}$, if $z \in P\left(z^{1} ; \overrightarrow{z^{2}}\right)$ then $\exists z^{\prime} \in P\left(\overrightarrow{z^{1}} ; \overrightarrow{z^{2}}\right)$ such that $z^{\prime} \preceq_{G L} z$.

Proof of Lemma 3. Let $z^{1} \neq \overrightarrow{z^{1}}$ (Otherwise, the result is immediate). Let $h$ be the minimum value for which $z_{h}^{1}>z_{h+1}^{1}$ holds. Define $z^{1 \prime}$ as the permutation obtained from $z^{1}$ by swapping $z_{h}^{1}$ and $z_{h+1}^{1}$. That is, $z^{1}=\left(z_{1}^{1}, z_{2}^{1}, \ldots, z_{h}^{1}, z_{h+1}^{1}, \ldots, z_{p}^{1}\right)$ and $z^{1 \prime}=$ $\left(z_{1}^{1}, z_{2}^{1}, \ldots, z_{h+1}^{1}, z_{h}^{1}, \ldots, z_{p}^{1}\right)$ where $z_{h}^{1}>z_{h+1}^{1}$. We will first show the following holds:

If $z \in P\left(z^{1} ; \overrightarrow{z^{2}}\right)$ then $\exists z^{\prime} \in P\left(z^{1 \prime} ; \overrightarrow{z^{2}}\right)$ such that $z^{\prime} \preceq_{G L} z$.

Suppose for an arbitrary $0 \leq \mu \leq 1$ we have a point $z: z=\mu \overrightarrow{z^{2}}+$ $(1-\mu) z^{1}$, that is $z \in P\left(z^{1} ; \overrightarrow{z^{2}}\right)$. Define $z^{\prime} \in P\left(z^{1 \prime} ; \overrightarrow{z^{2}}\right): z^{\prime}=\mu \overrightarrow{z^{2}}+$ $(1-\mu) z^{1 \prime}$.

One can easily show that $z$ and $z^{\prime}$ have the same elements except the $h$ th and $h+1$ th elements, which are as follows:

$$
\begin{aligned}
& z_{h}=\mu \overrightarrow{z^{2}}{ }_{h}+(1-\mu) z_{h}^{1} ; \\
& z_{h+1}=\mu \vec{z}_{h+1}+(1-\mu) z_{h+1}^{1} ; \\
& z_{h}^{\prime}=\mu \vec{z}_{{ }_{h}}+(1-\mu) z_{h+1}^{1} ; \\
& z_{h+1}^{\prime}=\mu \vec{z}_{h+1}+(1-\mu) z_{h}^{1} .
\end{aligned}
$$

Note that $z^{\prime} \preceq_{G L} z$ if $\operatorname{Min}\left\{z_{h}^{\prime}, z_{h+1}^{\prime}\right\} \leq \operatorname{Min}\left\{z_{h}, z_{h+1}\right\}$ and $z_{h}^{\prime}+z_{h+1}^{\prime} \leq$ $z_{h}+z_{h+1}$ (See Lemma 2 above). Let us check (Recall that $z_{h}^{1}>z_{h+1}^{1}$ ):

$$
\begin{aligned}
& \operatorname{Min}\left\{z_{h}^{\prime}, z_{h+1}^{\prime}\right\}=\operatorname{Min}\left\{\mu{\overrightarrow{z^{2}}}_{h}+(1-\mu) z_{h+1}^{1}, \mu{\overrightarrow{z^{2}}}_{h+1}+(1-\mu) z_{h}^{1}\right\} \\
& =\mu \vec{z}_{h}{ }_{h}+(1-\mu) z_{h+1}^{1}=z_{h}^{\prime} .
\end{aligned}
$$

We do not know what $\operatorname{Min}\left\{z_{h}, z_{h+1}\right\}$ is, hence we will compare $z_{h}^{\prime}$ with both $z_{h}$ and $z_{h+1}$.

$$
\begin{aligned}
& z_{h}^{\prime}=\mu{\overrightarrow{z^{2}}}_{h}+(1-\mu) z_{h+1}^{1} \leq \mu{\overrightarrow{z^{2}}}_{h}+(1-\mu) z_{h}^{1}=z_{h} \\
& z_{h}^{\prime}=\mu{\overrightarrow{z^{2}}}_{h}+(1-\mu) z_{h+1}^{1} \leq \mu{\overrightarrow{z^{2}}}_{h+1}+(1-\mu) z_{h+1}^{1}=z_{h+1} . \text { Hence, }
\end{aligned}
$$

$\operatorname{Min}\left\{z_{h}^{\prime}, z_{h+1}^{\prime}\right\} \leq \operatorname{Min}\left\{z_{h}, z_{h+1}\right\}$

$$
\begin{aligned}
z_{h}+z_{h+1} & =\mu{\overrightarrow{z^{2}}}_{h}+(1-\mu) z_{h}^{1}+\mu{\overrightarrow{z^{2}}}_{h+1}+(1-\mu) z_{h+1}^{1} \\
& =\mu \overrightarrow{z^{2}}{ }_{h}+(1-\mu) z_{h+1}^{1}+\mu \overrightarrow{z^{2}}{ }_{h+1}+(1-\mu) z_{h}^{1} \\
& =z_{h}^{\prime}+z_{h+1}^{\prime} . \text { That is, }
\end{aligned}
$$

$z_{h}^{\prime}+z_{h+1}^{\prime} \leq z_{h}+z_{h+1}$

From A.1 and A.2 the conditions of Lemma 2 is satisfied so $z^{\prime} \preceq_{G L} z$. Since $\mu$ is arbitrary, this result is valid for every $z \in$ $P\left(z^{1} ; \overrightarrow{z^{2}}\right)$.

We showed that if $z \in P\left(z^{1} ; \overrightarrow{z^{2}}\right)$, then $\exists z^{\prime} \in P\left(z^{1 \prime} ; \overrightarrow{z^{2}}\right)$ such that $z^{\prime} \preceq_{G L} z$, where $z^{1 \prime}$ is the permutation obtained by a single swap of two consecutive elements of $z^{1}$ as defined above.

Note that any permutation of vector $z^{1}$ will result in $\overrightarrow{z^{1}}$ if we apply a finite number of such binary contiguous swaps. Starting from the first element which is higher than its consecutive element, these type of swaps will eventually result in $\overrightarrow{z^{1}}$. Hence, we have the following result:

For any $z^{2}, z^{1} \in \mathbb{R}^{p}$, if $z \in P\left(z^{1} ; \overrightarrow{z^{2}}\right)$ then $\exists z^{\prime} \in P\left(\overrightarrow{z^{1}} ; \overrightarrow{z^{2}}\right)$ such that $z^{\prime} \varliminf_{G L} z$.

Proposition 1. For any $z^{2}, z^{1} \in \mathbb{R}^{p}$, if $z \in P\left(z^{1} ; z^{2}\right)$ then $\exists z^{\prime} \in$ $P\left(\overrightarrow{z^{1}} ; \overrightarrow{z^{2}}\right)$ such that $z^{\prime} \preceq_{G L} z$.

Proof of Proposition 1. Let $z^{1}=\Pi^{s}\left(\overrightarrow{z^{1}}\right)$ and $z^{2}=\Pi^{q}\left(\overrightarrow{z^{2}}\right)$. Then $z=\mu\left(\Pi^{q}\left(\overrightarrow{z^{2}}\right)\right)+(1-\mu)\left(\Pi^{s}\left(\overrightarrow{z^{1}}\right)\right)$ for some $0 \leq \mu \leq 1$. Let the inverse permutation of $\Pi^{q}$ be $\Pi^{r}$ and let $\Pi^{r}\left(\Pi^{s}\right)=\Pi^{t}$.

We can rewrite the condition as follows: If $\Pi^{r}(z) \in$ $P\left(\Pi^{t}\left(\overrightarrow{z^{1}}\right) ; \overrightarrow{z^{2}}\right)$ then $\exists z^{\prime} \in P\left(\overrightarrow{z^{1}} ; \overrightarrow{z^{2}}\right)$ such that $z^{\prime} \preceq_{G L} \Pi^{r}(z)$, implied by Lemma 3 proved above.

Lemma 4. Every point in a k-point polyhedron is a convex combination of $k-1$ points which are in the $k-1$ distinct two-point polyhedrons generated by one of the upper generators and the lower generator. That is, given $z^{i}$ such that $z^{i} \succ z^{k}, \forall z^{i} \in R$ we have the following:

If $z \in P\left(R ; z^{k}\right)$ then there exists $\lambda_{i}$ and $y^{i} \in P\left(z^{i} ; z^{k}\right)$ for $i=$ $1, \ldots, k-1$ such that $z=\sum_{i=1}^{k-1} \lambda_{i} y^{i}$ and $\sum_{i=1}^{k-1} \lambda_{i}=1$.

Proof of Lemma 4. $z \in P\left(R ; z^{k}\right)$ hence $z=\mu z^{k}+\sum_{i=1}^{k-1} \mu_{i} z^{i}$ such that $\mu+\sum_{i=1}^{k-1} \mu_{i}=1$.

Let $y^{i}=\left(1-\mu_{i}^{\prime}\right) z^{k}+\mu_{i}^{\prime} z^{i} \forall i$.

Now we will show that there exist $\lambda_{i} i=1, \ldots, k-1$ such that $z=\sum_{i=1}^{k-1} \lambda_{i} y^{i}$. Given $\mu_{i}$ corresponding to vector $z$, we will show that $\lambda_{i}$ s and $\mu_{i}^{\prime} s$ exist as defined so that we can write $z$ as a convex combination of $y^{i}$ s. Suppose that we have $\lambda_{i}$ values for $i=1, \ldots, k-2$ such that $\lambda_{i}>0$ and $\sum_{i=1}^{k-2} \lambda_{i}<1$ and we set $\lambda_{k-1}=$ $1-\sum_{i=1}^{k-2} \lambda_{i}$. Given these $\lambda_{i}$ and $\mu_{i}$, we can set $\mu_{i}^{\prime}$ values as follows:

$$
\begin{aligned}
& \mu_{i}=\lambda_{i} \mu_{i}^{\prime} \text { for } i=1, \ldots, k-1 \\
& \mu_{i}^{\prime}=\mu_{i} / \lambda_{i} . \text { Since } \lambda_{i}>0 \text { and } \mu_{i} \geq 0, \text { we have } \mu_{i}^{\prime} \geq 0 .
\end{aligned}
$$

$$
\begin{aligned}
z & =\mu z^{k}+\sum_{i=1}^{k-1} \mu_{i} z^{i} \\
& =\left(1-\sum_{i=1}^{k-1} \lambda_{i} \mu_{i}^{\prime}\right) z^{k}+\sum_{i=1}^{k-1} \lambda_{i} \mu_{i}^{\prime} z^{i} \text { (Since } \mu=1-\sum_{i=1}^{k-1} \mu_{i}
\end{aligned}
$$




$$
\begin{aligned}
& \left.=1-\sum_{i=1}^{k-1} \lambda_{i} \mu_{i}^{\prime}\right) \\
& =\sum_{i=1}^{k-1} \lambda_{i}\left(1-\mu_{i}^{\prime}\right) z^{k}+\sum_{i=1}^{k-1} \lambda_{i} \mu_{i}^{\prime} z^{i} . \\
& =\sum_{i=1}^{k-1} \lambda_{i}\left[\left(1-\mu_{i}^{\prime}\right) z^{k}+\mu_{i}^{\prime} z^{i}\right] \\
& =\sum_{i=1}^{k-1} \lambda_{i} y^{i}
\end{aligned}
$$

Note that Lemma 4 can be expressed more sharply as follows: Every point in a $k$-point polyhedron is a convex combination of $k-1$ points which are in the $k-1$ distinct two-point polyhedrons, each of which is generated by one of the generators (which is used in all these two-point polyhedrons) and each one of the other generators. The above proof mechanism can be used to prove it: replace $z^{k}$ with an arbitrary generator and repeat the steps.

Proof of Theorem 7 We claim that if $z \in U\left(z^{k}\right)$ then $z \in \hat{U}\left(z^{k}\right)$.

Proof of Theorem 7. If $z \in U\left(z^{k}\right)$ then there exists $z^{\prime} \in P\left(\Pi\left(R \cup z^{k}\right)\right.$; $\left.\Pi^{s}\left(z^{k}\right)\right): z^{\prime} \preceq_{G L} z$ for some permutation $\Pi^{s}\left(z^{k}\right)$ of $z^{k}$. First note that this polyhedron includes all permutations of $z^{k}$, so without loss of generality we can assume $\Pi^{s}\left(z^{k}\right)=z^{k}$ as the lower generator.

Note that $R$ is the set of reference distributions each of which is preferred to the lower generator $z^{k}$. For the sake of simplicity and with an abuse of notation, in this proof we are going to refer to $\Pi\left(R \cup z^{k}\right)$ as $R$ (this is again without loss of generality).

From Lemma 4 if $z^{\prime} \in P\left(R ; z^{k}\right)$ then we can find $y^{i} \in P\left(z^{i} ; z^{k}\right)$ : $z^{\prime}=\sum_{i=1}^{k-1} \lambda_{i} y^{i}$ for $i=1,2, \ldots, k-1$, where $\sum_{i=1}^{k-1} \lambda_{i}=1$.

By Proposition 1, $y^{i} \in P\left(z^{i} ; z^{k}\right) \Rightarrow \exists z^{\prime \prime} \in P\left(\overrightarrow{z^{i}} ; \overrightarrow{z^{k}}\right)$ (hence $z^{\prime \prime} \in$ $\left.P\left(\vec{R} ; \overrightarrow{z^{k}}\right)\right): z^{\prime \prime} \preceq_{G L} y^{i}$ for all $y^{i} i=1,2, \ldots, k-1$. Since $y^{i} s$, as defined above, are all in set $\left\{z^{\prime}: z^{\prime \prime} \preceq_{G L} z^{\prime}\right.$ for some $\left.z^{\prime \prime} \in P\left(\vec{R} ; \overrightarrow{z^{k}}\right)\right\}$ and this set is convex; any convex combination of them will also be in the same set. Hence, $\exists z^{\prime \prime} \in P\left(\vec{R} ; \overrightarrow{z^{k}}\right): z^{\prime \prime} \preceq_{G L} z^{\prime}$.

To summarise, if $z^{\prime} \in P\left(\Pi\left(R \cup z^{k}\right) ; z^{k}\right)$ then $\exists z^{\prime \prime} \in P\left(\vec{R} ; \overrightarrow{z^{k}}\right)$ : $z^{\prime \prime} \preceq_{G L} z^{\prime}$ (i.e., we can use the ordered vectors of the generators only). Then we have $z^{\prime \prime} \in P\left(\vec{R} ; \overrightarrow{z^{k}}\right): z^{\prime \prime} \preceq_{G L} z$ (by transitivity). Recall that $\hat{U}\left(z^{k}\right)=\left\{z \mid z^{\prime} \preceq_{G L} z\right.$ for some $\left.z^{\prime} \in P\left(\vec{R} ; \overrightarrow{z^{k}}\right)\right\}$. Hence if $z \in U\left(z^{k}\right)$ then $z \in \hat{U}\left(z^{k}\right)$.

\section{A.1.2. Proof of Theorem 8}

In this part we are going to prove the rest of Theorem 5 on the equivalence of using $L\left(z^{k}\right)$ and $\hat{L}\left(z^{k}\right) . L\left(z^{k}\right)$ is the union of cdominated regions of all permutation cones generated using all the permutations of the generators. For a distribution to be in $L\left(z^{k}\right)$, it is sufficient if it belongs to the dominated region of any of these permutation cones.

The following theorem is our main result on $L\left(z^{k}\right)$.

Theorem 8. If $z \in L\left(z^{k}\right)$ then $z \in \hat{L}\left(z^{k}\right)$.

Given preference information $\preceq_{R}\left(z^{i} \succ z^{k}\right.$ for $\left.z^{i} \in R\right)$, for each permutation of $\overrightarrow{z^{k}}$, say $\Pi^{s}\left(\overrightarrow{z^{k}}\right)$, we can generate a permutation cone of the form

$C\left(\Pi^{1}\left(\overrightarrow{z^{1}}\right), \ldots, \Pi^{p !}\left(\overrightarrow{z^{1}}\right), \ldots, \Pi^{1}\left(\overrightarrow{z^{k-1}}\right), \ldots, \Pi^{p !}\left(\overrightarrow{z^{k-1}}\right)\right.$,

$\left.\Pi^{1}\left(\overrightarrow{z^{k}}\right), \ldots, \Pi^{p !}\left(\overrightarrow{z^{k}}\right) ; \Pi^{s}\left(\overrightarrow{z^{k}}\right)\right)$

This cone has all the permutations of the upper generators, $z^{i} \in R$. Note that it also has all permutations of the lower generator $\Pi^{s}\left(\overrightarrow{z^{k}}\right)$ as upper generators since any permutation of a distribution is weakly preferred to itself by symmetry and can be considered as an upper generator. For notational simplicity we denote this cone as $C\left(\Pi\left(R \cup z^{k}\right) ; \Pi^{s}\left(z^{k}\right)\right)$.

This is the largest cone that we can generate for $\Pi^{s}\left(\overrightarrow{z^{k}}\right)$ as the lower generator given this preference information. We have $p$ ! such cones each having a different permutation of $\overrightarrow{z^{k}}$ as the lower generator. The region we are interested in, $L\left(z^{k}\right)$, is the union of the c-dominated regions of these $p$ ! cones, $L\left(z^{k}\right)=\left\{z \mid z \preceq_{G L} z^{\prime}\right.$ for some $z^{\prime} \in C(\Pi(R \cup$ $\left.\left.z^{k}\right) ; \Pi^{s}\left(z^{k}\right)\right)$ for some permutation $\Pi^{s}\left(z^{k}\right)$ of $\left.z^{k}\right\}$.

A short sketch of the proof is as follows:

1. We first show in Remark 1 that these $p$ ! cones have the same c-dominated region since they are reflections of each other. Hence it is sufficient to use only one of them. For convenience, we use $C\left(\Pi\left(R \cup z^{k}\right) ; \overrightarrow{z^{k}}\right)$.

2. $C\left(\Pi\left(R \cup z^{k}\right) ; \overrightarrow{z^{k}}\right)$ is a convex region defined by an extreme point $\left(\overrightarrow{z^{k}}\right)$ and extreme rays. In Lemma 5 we determine nonextreme rays of the region and state this result in Corollary 2.

3. Finally in Theorem 8 we link the above results together. We now give the detailed results.

Remark 1. Sets Set $1=\left\{z: z \preceq_{G L} z^{\prime}\right.$ and $\left.z^{\prime} \in C\left(\Pi\left(R \cup z^{k}\right) ; \overrightarrow{z^{k}}\right)\right\}$ and Set $2=\left\{z: z \preceq_{G L} z^{\prime}\right.$ and $\left.z^{\prime} \in C\left(\Pi\left(R \cup z^{k}\right) ; \Pi^{s}\left(\overrightarrow{z^{k}}\right)\right)\right\}$ are equivalent for any $\Pi^{s}\left(\overrightarrow{z^{k}}\right)$ of $\overrightarrow{z^{k}}$.

Proof of Remark 1. This proof consists of two parts. In the first part we will show that $\forall z \in \operatorname{Set} 1, z \in \operatorname{Set} 2$ and in the second part we will show that $\forall z \in \operatorname{Set} 2, z \in \operatorname{Set}$.

Part 1: Now we want to prove $\forall z \in \operatorname{Set} 1, z \in \operatorname{Set} 2$.

Set $1=\left\{z: z \preceq_{G L} z^{\prime}\right.$ and $\left.z^{\prime} \in C\left(\Pi\left(R \cup z^{k}\right) ; \overrightarrow{z^{k}}\right)\right\}$. The c-dominated region consists of distributions $z$, which are generalised Lorenz dominated by a point $z^{\prime}$ on the cone. Any point $z^{\prime}$ on the cone can be expressed by the following expression:

$$
z^{\prime}=\overrightarrow{z^{k}}+\sum_{j=1}^{p !} \sum_{i=1}^{k-1} \mu_{j i}\left(\overrightarrow{z^{k}}-\Pi^{j}\left(\overrightarrow{z^{i}}\right)\right)+\sum_{j=1}^{p !} \beta_{j}\left(\overrightarrow{z^{k}}-\Pi^{j}\left(\overrightarrow{z^{k}}\right)\right)
$$

for $\mu_{j i} \geq 0$ and $\beta_{j} \geq 0$ by definition.

$$
\text { Apply } \Pi^{s} \text { so that }
$$

$$
\Pi^{s}\left(z^{\prime}\right)=\Pi^{s}\left(\overrightarrow{z^{k}}\right)+\sum_{j=1}^{p !} \sum_{i=1}^{k-1} \mu_{j i}\left(\Pi^{s}\left(\overrightarrow{z^{k}}\right)-\Pi^{j}\left(\overrightarrow{z^{i}}\right)\right)+
$$

$\sum_{j=1}^{p !} \beta_{j}\left(\Pi^{s}\left(\overrightarrow{z^{k}}\right)-\Pi^{j}\left(\overrightarrow{z^{k}}\right)\right)$. We rearrange the elements of vector $z^{\prime}$ such that we obtain $\Pi^{s}\left(z^{\prime}\right)$. This rearranged vector $\Pi^{s}\left(z^{\prime}\right)$ is on the cone generated by the rearranged versions of the cone generators. As we consider all permutations of the upper generator vectors in $C\left(\Pi\left(R \cup z^{k}\right) ; \overrightarrow{z^{k}}\right)$, this rearrangement affects only the lower generator. That is, $\Pi^{s}\left(z^{\prime}\right) \in C\left(\Pi\left(R \cup z^{k}\right) ; \Pi^{s}\left(\overrightarrow{z^{k}}\right)\right)$ by definition. Since the generalised Lorenz dominance relation has symmetry property $z$ is generalised Lorenz dominated by any permutation of $z^{\prime}$, hence it is in Set2. That is,

$z \preceq_{G L} z^{\prime} \Rightarrow z \preceq_{G L} \Pi^{S}\left(z^{\prime}\right)$ hence $z \in$ Set 2 .

Part 2: We skip the explanations since the structure of the proof is the same as that of part 1 . Now we want to prove $\forall z \in \operatorname{Set} 2$, $z \in \operatorname{Set} 1$.

$$
\begin{gathered}
\text { Set } 2=\left\{z: z \preceq_{G L} z^{\prime} \text { and } z^{\prime} \in C\left(\Pi\left(R \cup z^{k}\right) ; \Pi^{s}\left(\overrightarrow{z^{k}}\right)\right)\right\} . \\
z^{\prime}=\Pi^{s}\left(\overrightarrow{z^{k}}\right)+\sum_{j=1}^{p !} \sum_{i=1}^{k-1} \mu_{j i}\left(\Pi^{s}\left(\overrightarrow{z^{k}}\right)-\Pi^{j}\left(\overrightarrow{z^{i}}\right)\right)+ \\
\sum_{j=1}^{p !} \beta_{j}\left(\Pi^{s}\left(\overrightarrow{z^{k}}\right)-\Pi^{j}\left(\overrightarrow{z^{k}}\right)\right) \text { for } \mu_{j i} \geq 0 \text { and } \beta_{j} \geq 0 .
\end{gathered}
$$

Let $\Pi^{r}$ be the inverse permutation of $\Pi^{s}$ (that is, $\Pi^{r}\left(\Pi^{s}(z)\right)=$ $z)$. Apply $\Pi^{r}$ so that

$$
\Pi^{r}\left(z^{\prime}\right)=\overrightarrow{z^{k}}+\sum_{j=1}^{p !} \sum_{i=1}^{k-1} \mu_{j i}\left(\overrightarrow{z^{k}}-\Pi^{j}\left(\overrightarrow{z^{i}}\right)\right)+\sum_{j=1}^{p !} \beta_{j}\left(\overrightarrow{z^{k}}-\right.
$$

$\left.\Pi^{j}\left(\overrightarrow{z^{k}}\right)\right)$. That is, $\left.\Pi^{r}\left(z^{\prime}\right) \in C\left(\Pi\left(R \cup z^{k}\right) ; \overrightarrow{z^{k}}\right)\right\}$. We have

$$
z \preceq_{G L} z^{\prime} \Rightarrow z \preceq_{G L} \Pi^{r}\left(z^{\prime}\right) \text { hence } z \in \operatorname{Set} 1 \text {. }
$$




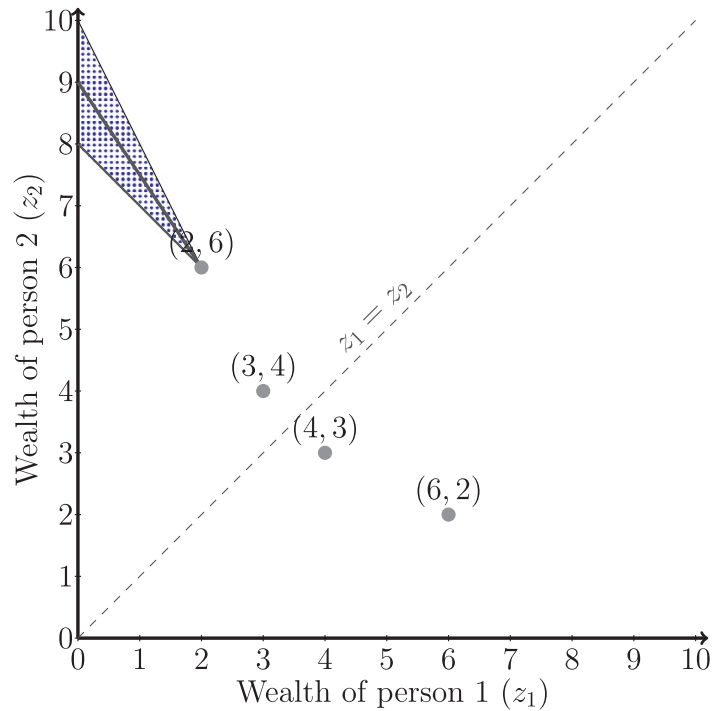

Fig. A5. Region $C((3,4),(4,3),(2,6),(6,2) ;(2,6))$.

Remark 1 implies that the c-dominated regions of all the cones of the form $C\left(\Pi\left(R \cup z^{k}\right) ; \Pi^{s}\left(\overrightarrow{z^{k}}\right)\right)$ obtained by using different permutations of the lower generator $z^{k}$ are the same. Hence it is sufficient to use only one of them. For convenience, we use $C(\Pi(R \cup$ $\left.z^{k}\right) ; \overrightarrow{z^{k}}$ ) and the corresponding dominated set Set1. Hence, Set 1 is a region that summarises all the information provided by all the permutation cones generated based on $z^{i} \succ z^{k}, z^{i} \in R$. Given preference information $\preceq_{R}$, for any distribution $z$, to check whether $z$ is in $L\left(z^{k}\right)$ we check whether $z$ is in Set 1 .

$C\left(\Pi\left(R \cup z^{k}\right) ; \overrightarrow{z^{k}}\right)$ is a convex set (cone), defined by an extreme point $\left(\overrightarrow{z^{k}}\right)$ and a set of rays generated as in Definition 5 below.

Definition 5. For $z^{i} \in \mathbb{R}^{p}: z^{i} \succ z^{k}$ for all $z^{i} \in R, C\left(\Pi\left(R \cup z^{k}\right) ; \overrightarrow{z^{k}}\right)=$ $\overrightarrow{z^{k}}+\sum \lambda_{t} r_{t}$ such that $\lambda_{t} \geq 0$ and $r_{t}$ are the rays in set Setofrays $=$ \{Rays defined by $\left(\overrightarrow{z^{k}}-\Pi^{j}\left(\overrightarrow{z^{i}}\right)\right)$, for all $z^{i} \in R$ and $j=1, \ldots, p$ ! , rays defined by $\left(\overrightarrow{z^{k}}-\Pi^{j}\left(\overrightarrow{z^{k}}\right)\right.$ ) for all $\left.j=1, \ldots, p !\right\}$. (We assume that the vectors have been perturbed so that all entities of $z^{k}$ are nonidentical).

$C\left(\Pi\left(R \cup z^{k}\right) ; \overrightarrow{z^{k}}\right)$ can actually be characterised by the extreme point $\left(\overrightarrow{z^{k}}\right)$ and the extreme rays in set Setofrays. We will show that these extreme rays have a particularly simple structure. We claim in Lemma 5 that in region $C\left(\Pi\left(R \cup z^{k}\right) ; \overrightarrow{z^{k}}\right)$, the rays given by $z^{k}-\Pi^{j}\left(\overrightarrow{z^{i}}\right)$ for $z^{i} \in R$, where $\Pi^{j}\left(\overrightarrow{z^{i}}\right) \neq \overrightarrow{z^{i}}$ are not extreme, hence can be written as a nonnegative combination of the other rays in $R$. In other words the cones $C\left(\Pi^{j}\left(\overrightarrow{z^{i}}\right) ; \overrightarrow{z^{k}}\right): \Pi^{j}\left(\overrightarrow{z^{i}}\right) \neq \overrightarrow{z^{i}}$ do not lie on the boundary of the region $C\left(\Pi\left(R \cup z^{k}\right) ; \overrightarrow{z^{k}}\right)$. In our 2D example (recall that the preference statement by the SP is that $(3,4)$ is preferred to $(2,6))$ this corresponds to claiming that the ray $(2,6)-$ $(4,3)$, i.e. $(-2,3)$, is not an extreme ray for $C\left(\Pi\left(R \cup z^{k}\right) ; \overrightarrow{z^{k}}\right)$ (dotted region) and this is clearly seen in Fig. A.5.

Lemma 5. Part (i): In set Setofrays the rays given by $\overrightarrow{z^{k}}-\Pi^{j}\left(\overrightarrow{z^{i}}\right)$, where $\Pi^{j}\left(\overrightarrow{z^{i}}\right) \neq \overrightarrow{z^{i}}$, can be written as a nonnegative combination of the rays $\overrightarrow{z^{k}}-\overrightarrow{z^{i}}$ and $\overrightarrow{z^{k}}-\Pi^{j}\left(\overrightarrow{z^{k}}\right) \forall j: \Pi^{j}\left(\overrightarrow{z^{k}}\right)$ is obtained from $\overrightarrow{z^{k}}$ by swapping two consecutive elements (note that we have $p-1$ such $\left.\Pi^{j}\left(\overrightarrow{z^{k}}\right) s\right)$.
Part (ii): In set Setofrays the rays given by $\overrightarrow{z^{k}}-\Pi^{l}\left(\overrightarrow{z^{k}}\right)$ can be written as a nonnegative combination of the rays $\overrightarrow{z^{k}}-\Pi^{j}\left(\overrightarrow{z^{k}}\right) \forall j$ : $\Pi^{j}\left(\overrightarrow{z^{k}}\right)$ is obtained by swapping two consecutive elements.

Proof of Lemma 5. We will prove this for an arbitrary element $z^{i}$ $\in R$.

Let $z^{i} \neq \overrightarrow{z^{i}}$ as assumed. Let $h$ be the minimum value for which $z_{h}^{i}>z_{h+1}^{i}$ holds. Define $S\left(z^{i}\right)$ as the permutation obtained from $z^{i}$ by swapping $z_{h}^{i}$ and $z_{h+1}^{i}$ (i.e. $S$ is a swap operator). That is, $z^{i}=$ $\left(z_{1}^{i}, z_{2}^{i}, \ldots, z_{h}^{i}, z_{h+1}^{i}, \ldots, z_{p}^{i}\right)$ and $S\left(z^{i}\right)=\left(z_{1}^{i}, z_{2}^{i}, \ldots, z_{h+1}^{i}, z_{h}^{i}, \ldots, z_{p}^{i}\right)$ where $z_{h}^{i}>z_{h+1}^{i}$. Note that any permutation of vector $z^{i}$ will result in $\overrightarrow{z^{i}}$ if we apply a finite number of such binary contiguous swaps. Hence we will first show the following for a single swap.

$\overrightarrow{z^{k}}-z^{i}=\sum \lambda_{t} r_{t}$ where $\lambda_{t} \geq 0$ and $r_{t}$ are in the set $\left\{\overrightarrow{z^{k}}-S\left(z^{i}\right)\right.$ and $\overrightarrow{z^{k}}-\Pi^{j}\left(\overrightarrow{z^{k}}\right): \Pi^{j}\left(\overrightarrow{z^{k}}\right) \neq \overrightarrow{z^{k}}$ and $\Pi^{j}\left(\overrightarrow{z^{k}}\right)$ obtained by swapping the $h$ th and $h+1$ th elements of $\overrightarrow{z^{k}}$ for $h$ value used in the $S$ operator\}. That is, $\overrightarrow{z^{k}}-z^{i}$ can be written as a nonnegative combination of $\overrightarrow{z^{k}}-S\left(z^{i}\right)$ and $\overrightarrow{z^{k}}-\Pi^{j}\left(\overrightarrow{z^{k}}\right)\left(\Pi^{j}\left(\overrightarrow{z^{k}}\right)\right.$ as defined in Lemma 5).

For $z^{i}, S\left(z^{i}\right)$ as defined above the following holds:

$$
\overrightarrow{z^{k}}-z^{i}=\overrightarrow{z^{k}}-S\left(z^{i}\right)+\left(\frac{z_{h}^{i}-z_{h+1}^{i}}{\overrightarrow{z^{k}}{ }_{h+1}-\overrightarrow{z^{k}}{ }_{h}}\right)\left(\overrightarrow{z^{k}}-\Pi^{j}\left(\overrightarrow{z^{k}}\right)\right)
$$

where $\Pi^{j}\left(\overrightarrow{z^{k}}\right)_{i}={\overrightarrow{z^{k}}}_{i} \forall i \neq h, h+1$ and $\Pi^{j}\left(\overrightarrow{z^{k}}\right)_{h}={\overrightarrow{z^{k}}}_{h+1} ; \Pi^{j}\left(\overrightarrow{z^{k}}\right)_{h+1}$ $=\overrightarrow{z^{k}}{ }_{h}$ (all the elements of $\overrightarrow{z^{k}}$ are the same in $\Pi^{j}\left(\overrightarrow{z^{k}}\right)$ except for $h$ th and $h+1$ th being swapped and we have ${\overrightarrow{z^{k}}}_{h} \leq{\overrightarrow{z^{k}}}_{h+1}$ by definition).

It is clearly seen when we analyse the vectors in detail as below:

$$
\begin{aligned}
& \left(\begin{array}{c}
\overrightarrow{z^{k}}{ }_{1}-z_{1}^{i} \\
\cdots \\
{\overrightarrow{z^{k}}}_{h}-z_{h}^{i} \\
{\overrightarrow{z^{k}}}_{{ }_{h+1}}-z_{h+1}^{i} \\
\cdots \\
{\overrightarrow{z^{k}}}_{p}-z_{p}^{i}
\end{array}\right)=\left(\begin{array}{c}
{\overrightarrow{z^{k}}}_{1}-z_{1}^{i} \\
\cdots \\
{\overrightarrow{z^{k}}}_{h}-z_{h+1}^{i} \\
{\overrightarrow{z^{k}}}_{h+1}-z_{h}^{i} \\
\cdots \\
{\overrightarrow{z^{k}}}_{p}-z_{p}^{i}
\end{array}\right) \\
& +\left(\frac{z_{h}^{i}-z_{h+1}^{i}}{\overrightarrow{z^{k}}{ }_{h+1}-{\overrightarrow{z^{k}}}_{h}}\right)\left(\begin{array}{c}
{\overrightarrow{z^{k}}}_{1}-{\overrightarrow{z^{k}}}_{1} \\
{\overrightarrow{z^{k}}}_{h}-{\overrightarrow{z^{k}}}_{h+1} \\
{\overrightarrow{z^{k}}}_{h+1}-{\overrightarrow{z^{k}}}_{h} \\
{\overrightarrow{z^{k}}}_{p}-{\overrightarrow{z^{k}}}_{p}
\end{array}\right)
\end{aligned}
$$

In the above equation if $\overrightarrow{z^{k}}{ }_{h+1}>{\overrightarrow{z^{k}}}_{h}$ then $\left(\frac{z_{h}^{i}-z_{h+1}^{i}}{\overrightarrow{z^{k}}{ }_{h+1}-\vec{z}^{k}{ }_{h}}\right) \geq 0$, that is we are able to write the ray that corresponds to $\overrightarrow{z^{k}}-z^{i}$ as a nonnegative combination of the rays $\overrightarrow{z^{k}}-S\left(z^{i}\right)$ and $\overrightarrow{z^{k}}-\Pi^{j}\left(\overrightarrow{z^{k}}\right)$.

Recall that we assume that the vectors have been perturbed so that all entities of $z^{k}$ are nonidentical. That is, we do not have the following case: $\overrightarrow{z^{k}}{ }_{h+1}=\overrightarrow{z^{k}}{ }_{h}$, hence $\overrightarrow{z^{k}}=\Pi^{j}\left(\overrightarrow{z^{k}}\right)$.

At each such step we will be able to write the first ray $\left(\overrightarrow{z^{k}}-\right.$ $\left.z^{i}\right)$ as a nonnegative combination of the rays $\left(\overrightarrow{z^{k}}-S\left(z^{i}\right)\right.$ and $\overrightarrow{z^{k}}-$ $\left.\Pi^{j}\left(\overrightarrow{z^{k}}\right)\right)$. Starting from the first element which is higher than its consecutive element, these type of swaps will eventually result in $\overrightarrow{z^{i}}$. Hence, we have the following result: 
In set Setofrays the rays given by $\overrightarrow{z^{k}}-\Pi^{j}\left(\overrightarrow{z^{i}}\right)$, where $\Pi^{j}\left(\overrightarrow{z^{i}}\right) \neq$ $\overrightarrow{z^{i}}$ can be written as a nonnegative combination of the rays $\overrightarrow{z^{k}}-\overrightarrow{z^{i}}$ and $\overrightarrow{z^{k}}-\Pi^{j}\left(\overrightarrow{z^{k}}\right) \forall j: \Pi^{j}\left(\overrightarrow{z^{k}}\right)$ is obtained by making binary contagious swaps in $\overrightarrow{z^{k}}$ (note that we have $p-1$ such $\Pi^{j}\left(\overrightarrow{z^{k}}\right) s$ ).

Example 5. Below is an example case that shows how this proof works:

Suppose that $z^{i}=(3,2,1)$. First note that in a number of binary contagious swaps we can obtain $\overrightarrow{z^{i}}$ as follows:

$(3,2,1)$

Swap at $h=1$

$(2,3,1)$

swap 3 and 2

$(2,1,3)$

$$
\rightarrow
$$

Swap at $h=1$

Swap 2 and 1

Hence $S(3,2,1)=(2,3,1) ; S(2,3,1)=(2,1,3)$ and $S(2,1,3)=$ $(1,2,3)$.

Let us apply our result. Assume a generic $z^{k}$ vector. We have proven that:

$$
\overrightarrow{z^{k}}-z^{i}=\overrightarrow{z^{k}}-S\left(z^{i}\right)+\left(\frac{z_{h}^{i}-z_{h+1}^{i}}{\overrightarrow{z^{k}}{ }_{h+1}-{\overrightarrow{z^{k}}}_{h}}\right)\left(\overrightarrow{z^{k}}-\Pi^{j}\left(\overrightarrow{z^{k}}\right)\right) \text {, such that }
$$

$\Pi^{j}\left(\overrightarrow{z^{k}}\right)$ is obtained by swapping the $h$ th element with $h+1$ th element in $\overrightarrow{z^{k}}$. We see below that this holds for any $\overrightarrow{z^{k}}$.

Iteration 1: Start with $z^{i}=(3,2,1)$, hence $S\left(z^{i}\right)=(2,3,1), h=$ 1 and $\Pi^{j}\left(\overrightarrow{z^{k}}\right)=\left(\overrightarrow{z^{k}}{ }_{2}, \vec{z}^{k}{ }_{1}, \vec{z}^{k}{ }_{3}\right)$ obtained by swapping $1 s t$ (hth) and 2nd $\left(h+1\right.$ th) elements in $\overrightarrow{z^{k}}$.

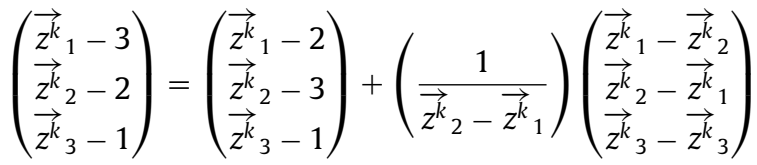

At row 1: ${\overrightarrow{z^{k}}}_{1}-3={\overrightarrow{z^{k}}}_{1}-2-1={\overrightarrow{z^{k}}}_{1}-3$

At row 2: $\vec{z}^{k}{ }_{2}-2={\overrightarrow{z^{k}}}_{2}-3+1={\overrightarrow{z^{k}}}_{2}-2$

At row 3: ${\overrightarrow{z^{k}}}_{3}-1={\overrightarrow{z^{k}}}_{3}-1+0={\overrightarrow{z^{k}}}_{3}-1$

Iteration 2: We set $z^{i}=(2,3,1)$ now. Hence $S\left(z^{i}\right)=(2,1,3)$, $h=2$, and $\Pi^{j}\left(\overrightarrow{z^{k}}\right)=\left(\overrightarrow{z^{k}}{ }_{1}, \vec{z}^{k}{ }_{3}, \vec{z}^{k}{ }_{2}\right)$ obtained by swapping $2 n d$ $\left(h\right.$ th) and $3^{\text {rd }}(h+1 t h)$ elements in $\overrightarrow{z^{k}}$.

$\left(\begin{array}{l}{\overrightarrow{z^{k}}}_{1}-2 \\ \overrightarrow{z^{k}}{ }_{2}-3 \\ \overrightarrow{z^{k}}{ }_{3}-1\end{array}\right)=\left(\begin{array}{l}{\overrightarrow{z^{k}}}_{1}-2 \\ \overrightarrow{z^{k}}{ }_{2}-1 \\ \overrightarrow{z^{k}}{ }_{3}-3\end{array}\right)+\left(\begin{array}{c}2 \\ \overrightarrow{z^{k}}{ }_{3}-\overrightarrow{z^{k}}\end{array}\right)\left(\begin{array}{l}\vec{z}_{2} \\ {\overrightarrow{z^{k}}}_{2}-{\overrightarrow{z^{k}}}_{1} \\ {\overrightarrow{z^{k}}}_{3} \\ \overrightarrow{z^{k}}{ }_{3}-{\overrightarrow{z^{k}}}_{2}\end{array}\right)$

At row 1: ${\overrightarrow{z^{k}}}_{1}-2={\overrightarrow{z^{k}}}_{1}-2-0={\overrightarrow{z^{k}}}_{1}-2$

At row 2: $\vec{z}^{k}{ }_{2}-3={\overrightarrow{z^{k}}}_{2}-1-2={\overrightarrow{z^{k}}}_{2}-3$

At row 3: $\vec{z}^{k}{ }_{3}-1={\overrightarrow{z^{k}}}_{3}-3+2={\overrightarrow{z^{k}}}_{3}-1$

Iteration 3: We set $z^{i}=(2,1,3)$ now. Hence $S\left(z^{i}\right)=(1,2,3)$, $h=1$, and $\Pi^{j}\left(\overrightarrow{z^{k}}\right)=\left(\overrightarrow{z^{k}}{ }_{2}, \vec{z}^{k}{ }_{1}, \vec{z}^{k}{ }_{3}\right)$ obtained by swapping $1 s t$ (hth) and $2^{\text {nd }}(h+1 s t)$ elements in $\overrightarrow{z^{k}}$.

$\left(\begin{array}{l}{\overrightarrow{z^{k}}}_{1}-2 \\ \overrightarrow{z^{k}}{ }_{2}-1 \\ \overrightarrow{z^{k}}{ }_{3}-3\end{array}\right)=\left(\begin{array}{l}{\overrightarrow{z^{k}}}_{1}-1 \\ \overrightarrow{z^{k}}{ }_{2}-2 \\ \overrightarrow{z^{k}}{ }_{3}-3\end{array}\right)+\left(\begin{array}{c}1 \\ \overrightarrow{z^{k}}{ }_{2}-\overrightarrow{z^{k}}{ }_{1}\end{array}\right)\left(\begin{array}{l}{\overrightarrow{z^{k}}}_{1}-{\overrightarrow{z^{k}}}_{2} \\ \overrightarrow{z^{k}}{ }_{2}-{\overrightarrow{z^{k}}}_{1} \\ \overrightarrow{z^{k}}{ }_{3}-{\overrightarrow{z^{k}}}_{3}\end{array}\right)$

At row $1: \vec{z}^{k}{ }_{1}-2={\overrightarrow{z^{k}}}_{1}-1-1=\overrightarrow{z^{k}}{ }_{1}-2$

At row 2: $\vec{z}_{2}-1={\overrightarrow{z^{k}}}_{2}-2+1={\overrightarrow{z^{k}}}_{2}-1$

At row 3: ${\overrightarrow{z^{k}}}_{3}-3={\overrightarrow{z^{k}}}_{3}-3+0={\overrightarrow{z^{k}}}_{3}-3$
To conclude

$$
\begin{aligned}
& \left(\begin{array}{l}
\overrightarrow{z^{k}}{ }_{1}-3 \\
\overrightarrow{z^{k}}{ }_{2}-2 \\
{\overrightarrow{z^{k}}}_{3}-1
\end{array}\right)=\left(\begin{array}{l}
{\overrightarrow{z^{k}}}_{1}-1 \\
{\overrightarrow{z^{k}}}_{2}-2 \\
{\overrightarrow{z^{k}}}_{3}-3
\end{array}\right)+\left(\begin{array}{c}
1 \\
\overrightarrow{z^{k}}{ }_{2}-{\overrightarrow{z^{k}}}_{1}
\end{array}\right)\left(\begin{array}{l}
{\overrightarrow{z^{k}}}_{1}-{\overrightarrow{z^{k}}}_{2} \\
{\overrightarrow{z^{k}}}_{2}-{\overrightarrow{z^{k}}}_{1} \\
\overrightarrow{z^{k}}{ }_{3}-{\overrightarrow{z^{k}}}_{3}
\end{array}\right) \\
& +\left(\frac{2}{\overrightarrow{z^{k}}{ }_{3}-\overrightarrow{z^{k}}}\right)\left(\begin{array}{l}
\overrightarrow{z^{k}} \\
\overrightarrow{z_{1}^{k}}-\overrightarrow{z^{k}}{ }_{1} \\
\overrightarrow{z^{k}}{\overrightarrow{z^{k}}}_{3}-{\overrightarrow{z^{k}}}_{2}
\end{array}\right) \\
& +\left(\frac{1}{\overrightarrow{z^{k}}{ }_{2}-\overrightarrow{z^{k}}}\right)\left(\begin{array}{l}
\overrightarrow{z^{k}} \\
\overrightarrow{z_{1}^{k}}-\overrightarrow{z^{k}} \overrightarrow{2}_{2}-{\overrightarrow{z^{k}}}_{1} \\
\overrightarrow{z^{k}}{ }_{3}-\overrightarrow{z^{k}}{ }_{3}
\end{array}\right)
\end{aligned}
$$

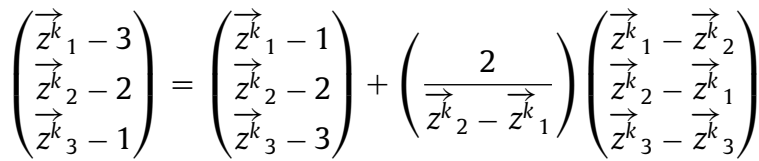

$$
\begin{aligned}
& +\left(\frac{2}{\overrightarrow{z^{k}}{ }_{3}-{\overrightarrow{z^{k}}}_{2}}\right)\left(\begin{array}{l}
\overrightarrow{z^{k}}{ }_{1}-{\overrightarrow{z^{k}}}_{1} \\
\overrightarrow{z^{k}} \overrightarrow{2}_{2}-{\overrightarrow{z^{k}}}_{3} \\
\overrightarrow{z^{k}}{ }_{3}-{\overrightarrow{z^{k}}}_{2}
\end{array}\right)
\end{aligned}
$$

At row 1: $\overrightarrow{z^{k}}{ }_{1}-3={\overrightarrow{z^{k}}}_{1}-1-2+0={\overrightarrow{z^{k}}}_{1}-3$

At row 2: $\vec{z}_{2}{ }_{2}-2={\overrightarrow{z^{k}}}_{2}-2+2-2={\overrightarrow{z^{k}}}_{2}-2$

At row 3: $\vec{z}^{k}{ }_{3}-1={\overrightarrow{z^{k}}}_{3}-3+0+2={\overrightarrow{z^{k}}}_{3}-1$

Since any permutation of $\overrightarrow{z^{i}}$ could be used at the beginning as $z^{i}$, this applies to any permutation.

The same proof mechanism can be used to show that this result holds for the rays $\overrightarrow{z^{k}}-\Pi^{l}\left(\overrightarrow{z^{k}}\right)$. That is, such rays can be written as a nonnegative combination of $\overrightarrow{z^{k}}-\Pi^{j}\left(\overrightarrow{z^{k}}\right): \forall \Pi^{j}\left(\overrightarrow{z^{k}}\right): \Pi^{j}\left(\overrightarrow{z^{k}}\right)$ is obtained by swapping two consecutive elements in $\overrightarrow{z^{k}}$. Simply set $z^{i}=\Pi^{l}\left(\overrightarrow{z^{k}}\right)$ and repeat the proof.

Corollary 2. In $C\left(\Pi\left(R \cup z^{k}\right) ; \overrightarrow{z^{k}}\right)$ the rays $\overrightarrow{z^{k}}-\Pi^{j}\left(\overrightarrow{z^{i}}\right): \Pi^{j}\left(\overrightarrow{z^{i}}\right) \neq$ $\overrightarrow{z^{i}}$ where $z^{i} \in R$ are not extreme rays. Also the rays $\overrightarrow{z^{k}}-\Pi^{j}\left(\overrightarrow{z^{k}}\right)$ : $\Pi^{j}\left(\overrightarrow{z^{k}}\right)$ cannot be obtained by swapping two consecutive elements in $\overrightarrow{z^{k}}$, are not extreme rays.

Proof of Corollary 2. By Lemma 5, these rays can be written in terms of the other rays in Setofrays. Hence they are not extreme rays of $C\left(\Pi\left(R \cup z^{k}\right) ; \overrightarrow{z^{k}}\right)$.

Proof of Theorem 8

If $z \in L\left(z^{k}\right)$ then $z \in \hat{L}\left(z^{k}\right)$, which is the set $\hat{L}\left(z^{k}\right)=\left\{z \mid z \preceq_{G L}\right.$ $z^{\prime}$ for some $\left.z^{\prime} \in C\left(\vec{R} \cup \hat{\Pi}\left(\overrightarrow{z^{k}}\right) ; \overrightarrow{z^{k}}\right)\right\}$, where $C\left(\vec{R} \cup \hat{\Pi}\left(\overrightarrow{z^{k}}\right) ; \overrightarrow{z^{k}}\right)=$ $\left\{z: z=\overrightarrow{z^{k}}+\sum_{i=1}^{k-1} \mu_{i}\left(\overrightarrow{z^{k}}-\overrightarrow{z^{i}}\right)+\sum_{j=1}^{p-1} \beta_{j}\left(\overrightarrow{z^{k}}-\Pi^{j}\left(\overrightarrow{z^{k}}\right)\right)\right.$, where $\mu_{i} \geq 0, \beta_{j} \geq 0, j: \Pi^{j}\left(\overrightarrow{z^{k}}\right)$ is obtained by swapping two consecutive elements in $\overrightarrow{z^{k}}$.

Proof of Theorem 8. $z \in L\left(z^{k}\right)$, that is in set $\left\{z \mid z \preceq_{G L} z^{\prime}\right.$ for some $z^{\prime} \in C\left(\Pi\left(R \cup z^{k}\right) ; \Pi^{s}\left(z^{k}\right)\right)$ for some permutation $\Pi^{s}\left(z^{k}\right)$ of $z^{k}$ \} and hence $z \in \operatorname{Set} 1=\left\{z: z \preceq_{G L} z^{\prime}\right.$ and $\left.z^{\prime} \in C\left(\Pi\left(R \cup z^{k}\right) ; \overrightarrow{z^{k}}\right)\right\}$ due to Remark 1. $C\left(\Pi\left(R \cup z^{k}\right) ; \overrightarrow{z^{k}}\right)$ is a convex region, defined by an extreme point $\left(\overrightarrow{z^{k}}\right)$ and a set of rays Setofrays generated as in Definition 5. By Corollary 2 we can exclude the non-extreme rays from the definition and hence use $C\left(\left(\vec{R} \cup \hat{\Pi}^{s}\left(z^{k}\right)\right) ; \overrightarrow{z^{k}}\right)=\{z$ : $z=\overrightarrow{z^{k}}+\sum_{i=1}^{k-1} \mu_{i}\left(\overrightarrow{z^{k}}-\overrightarrow{z^{i}}\right)+\sum_{j=1}^{p-1} \beta_{j}\left(\overrightarrow{z^{k}}-\Pi^{j}\left(\overrightarrow{z^{k}}\right)\right)$, where $\mu_{i} \geq 0$, 
$\beta_{j} \geq 0, j: \Pi^{j}\left(\overrightarrow{z^{k}}\right)$ is obtained by swapping two consecutive elements in $\left.\overrightarrow{z^{k}}\right\}$.

\section{A.2. The results for setting when one uses two-point cones}

\section{A.2.1. Proof of Theorem 6}

We first discuss Lemma 6 that will be used in our main proof of Theorem 6 .

Lemma 6. Given $z^{1}, z^{2} \in \mathbb{R}^{p}$, if $z \in C\left(z^{1} ; \overrightarrow{z^{2}}\right)$ then $\exists z^{\prime} \in C\left(\overrightarrow{z^{1}} ; \overrightarrow{z^{2}}\right)$ : $z \preceq_{G L} z^{\prime}$ (i.e. $z$ is generalised Lorenz dominated by a point in $\left.C\left(\overrightarrow{z^{1}} ; \overrightarrow{z^{2}}\right)\right)$.

Proof of Lemma 6. Let $z^{1} \neq \overrightarrow{z^{1}}$ (Otherwise, the result is immediate). Let $h$ be the minimum value for which $z_{h}^{1}>z_{h+1}^{1}$ holds. Define $z^{1 \prime}$ as the permutation obtained from $z^{1}$ by swapping $z_{h}^{1}$ and $z_{h+1}^{1}$. We will show the following holds:

If $z \in C\left(z^{1} ; \overrightarrow{z^{2}}\right)$ then $\exists z^{\prime} \in C\left(z^{1 \prime} ; \overrightarrow{z^{2}}\right): z \preceq_{G L} z^{\prime}$.

Suppose for an arbitrary $\mu \geq 0$ we have a point $z: z=\overrightarrow{z^{2}}+$ $\mu\left(\overrightarrow{z^{2}}-z^{1}\right)$, that is $z \in C\left(z^{1} ; \overrightarrow{z^{2}}\right)$. Define $z^{\prime} \in C\left(z^{1 \prime} ; \overrightarrow{z^{2}}\right): z^{\prime}=\overrightarrow{z^{2}}+$ $\mu\left(\overrightarrow{z^{2}}-z^{1 \prime}\right)$.

One can easily show that $z$ and $z^{\prime}$ have the same elements except the $h$ th and $h+1$ th elements, which are as follows:

$$
\begin{aligned}
& z_{h}={\overrightarrow{z^{2}}}_{h}+\mu\left(\overrightarrow{z^{2}}{ }_{h}-z_{h}^{1}\right) ; \\
& z_{h+1}=\overrightarrow{z^{2}}{ }_{h+1}+\mu\left(\vec{z}^{2}{ }_{h+1}-z_{h+1}^{1}\right) ; \\
& z_{h}^{\prime}=\vec{z}^{2}{ }_{h}+\mu\left(\vec{z}^{2}{ }_{h}-z_{h+1}^{1}\right) ; \\
& z_{h+1}^{\prime}=\vec{z}_{h+1}+\mu\left(\vec{z}^{2}{ }_{h+1}-z_{h}^{1}\right)
\end{aligned}
$$

From Lemma 2 we know that $z \preceq_{G L} z^{\prime}$ if $\operatorname{Min}\left\{z_{h}, z_{h+1}\right\} \leq$ $\operatorname{Min}\left\{z_{h}^{\prime}, z_{h+1}^{\prime}\right\}$ and $z_{h}+z_{h+1} \leq z_{h}^{\prime}+z_{h+1}^{\prime}$. Let us check (Recall that $\left.z_{h}^{1}>z_{h+1}^{1}\right)$ :

$$
\begin{aligned}
\operatorname{Min}\left\{z_{h}, z_{h+1}\right\}= & \operatorname{Min}\left\{\left[{\overrightarrow{z^{2}}}_{h}+\mu\left({\overrightarrow{z^{2}}}_{h}-z_{h}^{1}\right)\right],\right. \\
& {\left.\left[{\overrightarrow{z^{2}}}_{h+1}+\mu\left({\overrightarrow{z^{2}}}_{h+1}-z_{h+1}^{1}\right)\right]\right\} } \\
= & {\overrightarrow{z^{2}}}_{h}+\mu\left({\overrightarrow{z^{2}}}_{h}-z_{h}^{1}\right)=z_{h} .
\end{aligned}
$$

We do not know what $\operatorname{Min}\left\{z_{h}^{\prime}, z_{h+1}^{\prime}\right\}$ is, hence we will compare $z_{h}$ with both $z_{h}^{\prime}$ and $z_{h+1}^{\prime}$.

$$
\begin{aligned}
& z_{h}={\overrightarrow{z^{2}}}_{h}+\mu\left({\overrightarrow{z^{2}}}_{h}-z_{h}^{1}\right) \leq{\overrightarrow{z^{2}}}_{h}+\mu\left({\overrightarrow{z^{2}}}_{h}-z_{h+1}^{1}\right)=z_{h}^{\prime} \\
& z_{h}={\overrightarrow{z^{2}}}_{h}+\mu\left({\overrightarrow{z^{2}}}_{h}-z_{h}^{1}\right) \leq \vec{z}^{2}{ }_{h+1}+\mu\left(\vec{z}_{h+1}-z_{h}^{1}\right)=z_{h+1}^{\prime} \text {. Hence, }
\end{aligned}
$$

$$
\begin{aligned}
\operatorname{Min}\left\{z_{h}, z_{h+1}\right\} & \leq \operatorname{Min}\left\{z_{h}^{\prime}, z_{h+1}^{\prime}\right\} \\
z_{h}+z_{h+1} & =\vec{z}^{2}{ }_{h}+\mu\left({\overrightarrow{z^{2}}}_{h}-z_{h}^{1}\right)+{\overrightarrow{z^{2}}}_{h+1}+\mu\left({\overrightarrow{z^{2}}}_{h+1}-z_{h+1}^{1}\right) \\
& =z_{h+1}^{\prime}+z_{h}^{\prime} . \text { That is, } \\
z_{h}+z_{h+1} \leq & z_{h}^{\prime}+z_{h+1}^{\prime}
\end{aligned}
$$

From A.3 and A.4 the conditions of Lemma 2 are satisfied so $z \preceq_{G L} z^{\prime}$. Since $\mu$ is arbitrary, this result is valid for every $z \in$ $C\left(z^{1} ; \overrightarrow{z^{2}}\right)$.

We showed that if $z \in C\left(z^{1} ; \overrightarrow{z^{2}}\right)$, then $\exists z^{\prime} \in C\left(z^{1 \prime} ; \overrightarrow{z^{2}}\right): z \preceq_{G L} z^{\prime}$. where $z^{1 \prime}$ is the permutation obtained by a single swap of two consecutive elements of $z^{1}$ as defined above. Note that any permutation of vector $z^{1}$ will result in $\overrightarrow{z^{1}}$ if we apply a finite number of such binary contiguous swaps. Starting from the first element which is higher than its consecutive element, these type of swaps will eventually result in $\overrightarrow{z^{1}}$. Hence, we have the following result:

For any $z^{1}, z^{2} \in \mathbb{R}^{p}$, if $z \in C\left(z^{1} ; \overrightarrow{z^{2}}\right)$ then $\exists z^{\prime} \in C\left(\overrightarrow{z^{1}} ; \overrightarrow{z^{2}}\right): z \preceq_{G L}$

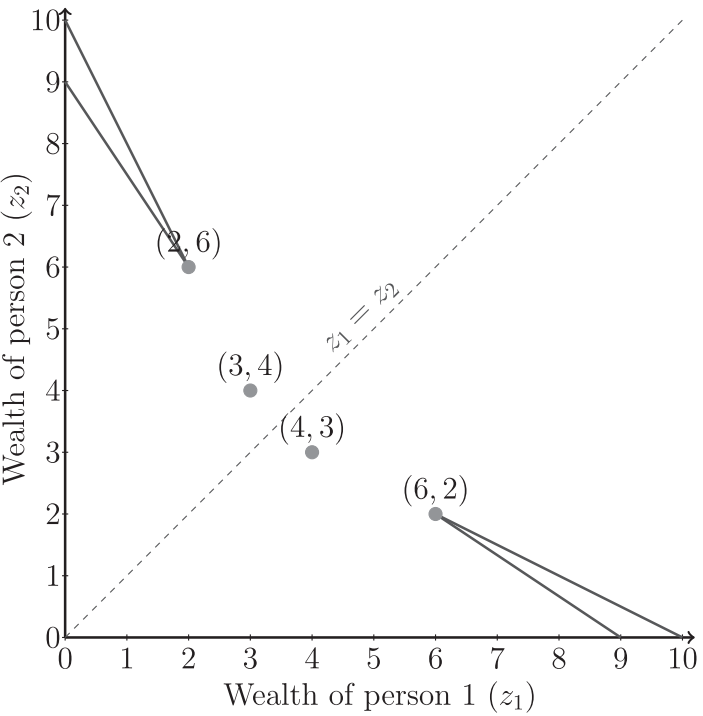

Fig. A6. Permutation cones in $\mathbb{R}^{2}$.

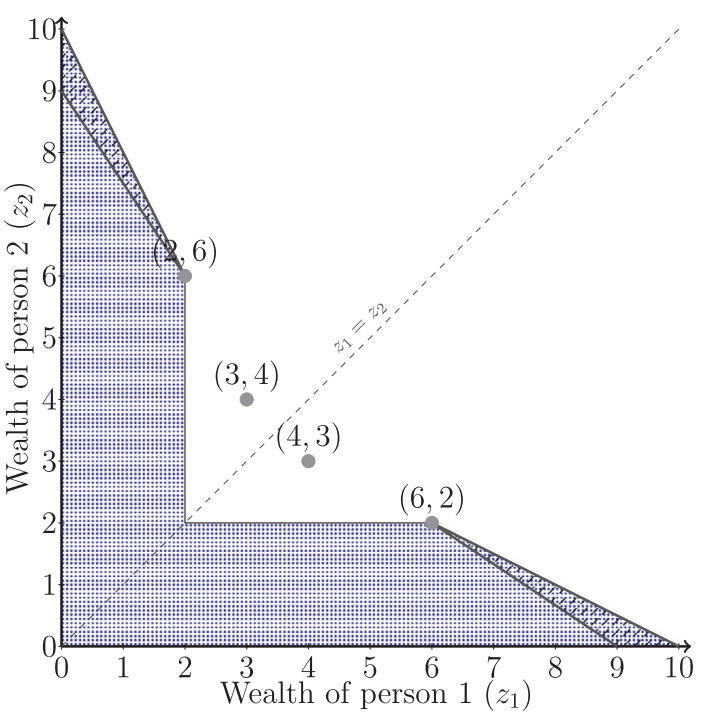

Fig. A7. $\bar{L}((2,6))$.

Proof of Theorem 6

The conclusion that for any distribution $z$ if $z \in U\left(z^{k}\right)$ then $z \in$ $\hat{U}\left(z^{k}\right)$ is by Theorem 5 . We are going to show that the remaining part of Theorem 6 holds, i.e., if $z \in L\left(z^{k}\right)$ then $z \in \bar{L}\left(z^{k}\right)$. That is for any $z^{i}, z^{k}, z \in \mathbb{R}^{p}$, if $z \in L\left(z^{k}\right)$ there exists a $z^{\prime \prime} \in C\left(\overrightarrow{z^{i}} ; \overrightarrow{z^{k}}\right): z \preceq_{G L} z^{\prime \prime}$.

Proof of Theorem 6. In this special case $L\left(z^{k}\right)$ is the union of the c-dominated regions of all cones of the form $C\left(\Pi^{r}\left(z^{i}\right) ; \Pi^{s}\left(z^{k}\right)\right)$ for any $r$ and s. If $z \in L\left(z^{k}\right)$ there exists a $z^{\prime} \in C\left(\Pi^{r}\left(\overrightarrow{z^{i}}\right) ; \Pi^{s}\left(\overrightarrow{z^{k}}\right)\right)$ for some permutations $r$ and $s$ such that $z \preceq_{G L} z^{\prime}$. Hence $z^{\prime}=\Pi^{s}\left(\overrightarrow{z^{k}}\right)+$ $\mu\left(\Pi^{s}\left(\overrightarrow{z^{k}}\right)-\Pi^{r}\left(\overrightarrow{z^{i}}\right)\right)$ for some $\mu \geq 0$. Let the inverse permutation of $\Pi^{s}$ be $\Pi^{q}$ and let $\Pi^{q}\left(\Pi^{r}\right)=\Pi^{t}$. Then $\Pi^{q}\left(z^{\prime}\right) \in C\left(\Pi^{t}\left(\overrightarrow{z^{i}}\right) ; \overrightarrow{z^{k}}\right)$.

If $\Pi^{q}\left(z^{\prime}\right) \in C\left(\Pi^{t}\left(\overrightarrow{z^{i}}\right) ; \overrightarrow{z^{k}}\right)$ then $\exists z^{\prime \prime} \in C\left(\overrightarrow{z^{i}} ; \overrightarrow{z^{k}}\right): \Pi^{q}\left(z^{\prime}\right) \preceq_{G L} z^{\prime \prime}$ $\forall t$, implied by Lemma 6 proved above. Then from transitivity, $z \preceq_{G L}$ $z^{\prime} \preceq_{G L} z^{\prime \prime}$. Hence $z \in \bar{L}\left(z^{k}\right)$.

This result is easily observed in Figs. A.6 and A.7 for $p=2$ (in $\mathbb{R}^{2}$ ). Fig. A.6 shows the 4 two-point cones based on the preference information $(3,4) \succ(2,6)$, where each line shows a cone. Fig. A.7 shows the set $\bar{L}(2,6)$. One 
can verify by simple observation that $\bar{L}((2,6))=\left\{z \mid z \preceq_{G L}\right.$ $z^{\prime}$ for some $\left.z^{\prime} \in C((3,4) ;(2,6))\right\}=\left\{z \mid z \preceq_{G L} z^{\prime}\right.$ for some $z^{\prime} \in$ $C((4,3) ;(6,2))\} \supset\left\{z \mid z \preceq_{G L} z^{\prime}\right.$ for some $\left.z^{\prime} \in C((4,3) ;(2,6))\right\}=$ $\left\{z \mid z \preceq_{G L} z^{\prime}\right.$ for some $\left.z^{\prime} \in C((3,4) ;(6,2))\right\}$. The dotted region is $\left\{z \mid z \preceq_{G L} z^{\prime}\right.$ for some $\left.z^{\prime} \in C((4,3) ;(2,6))\right\}$ and the region with diagonal lines is $\left\{z \mid z \preceq_{G L} z^{\prime}\right.$ for some $z^{\prime} \in C((3,4)$; $\left.(2,6))\right\} \backslash\left\{z \mid z \preceq_{G L}\right.$ $z^{\prime}$ for some $\left.z^{\prime} \in C((4,3) ;(2,6))\right\}$.

\section{A.2.2. Counterexample showing that Theorem 6 does not apply to} larger cones

Example 6. Suppose that we have a case where $k=3$ and $p=3$, that is we have three-point cones and we work in $\mathbb{R}^{3}$. Suppose that the SP has the following evaluation function:

$f(x)=x_{1} x_{2} x_{3}$.

Suppose that we present the following distributions to the SP for him to compare:

$z^{1}=(25,4,15)$

$z^{2}=(7,11,27)$

$z^{3}=(6,7,33)$

The corresponding utility values are $f\left(z^{1}\right)=1500, f\left(z^{2}\right)=$ 2079, $f\left(z^{3}\right)=1386$. Hence the SP will provide us with the information that $z^{2} \succ z^{3}$ and $z^{1} \succ z^{3}$. Based on this we can generate the corresponding 3-point cones.

We will show that there exists a point $z: z \in C\left(z^{1}, z^{2} ; z^{3}\right)$ (therefore $\left.z \in L\left(z^{3}\right)\right)$ and $\nexists z^{\prime} \in C\left(\overrightarrow{z^{1}}, \overrightarrow{z^{2}} ; \overrightarrow{z^{3}}\right): z \preceq_{G L} z^{\prime} . z=(4.82,4.65,37.2)$ is such an example.

$z \in C\left(z^{1}, z^{2} ; z^{3}\right)$ since $z=z^{3}+\sum_{i=1}^{2} \mu_{i}\left(z^{3}-z^{i}\right)$ where $\mu_{1}=0.03$ and $\mu_{2}=0.61$.

Let us check whether there is a $z^{\prime} \in C\left(\overrightarrow{z^{1}}, \overrightarrow{z^{2}} ; \overrightarrow{z^{3}}\right): z \preceq_{G L} z^{\prime}$. We solve the following LP:

Max 0

subject to

$z_{1}^{\prime}-2 \mu_{1}^{\prime}+1 \mu_{2}^{\prime}=6$

$z_{2}^{\prime}+8 \mu_{1}^{\prime}+4 \mu_{2}^{\prime}=7$

$z_{3}^{\prime}-8 \mu_{1}^{\prime}-6 \mu_{2}^{\prime}=33$

$r_{1}-d_{11}-d_{12}-d_{13} \geq 4.65$

$2 r_{2}-d_{21}-d_{22}-d_{23} \geq 9.47$

$3 r_{3}-d_{31}-d_{32}-d_{33} \geq 46.67$

$r_{n}-z_{i}^{\prime}-d_{n i} \leq 0 \quad i, n=1,2,3$

$\mu_{1}^{\prime}, \mu_{2}^{\prime} \geq 0$

$d_{n i} \geq 0 \quad i, n=1,2,3$

The above problem is infeasible, which shows that there is no $z^{\prime} \in C\left(\overrightarrow{z^{1}}, \overrightarrow{z^{2}} ; \overrightarrow{z^{3}}\right): z \preceq_{G L} z^{\prime}$.

\section{Appendix B. Checking whether a given distribution is in the $\mathbf{L}$ or $\mathbf{U}$}

\section{B.1. Checking whether a given distribution is in $L$}

Recall that by Theorem 8 if $z \in L\left(z^{k}\right)$ then there exists $z^{\prime} \in$ $C\left(\left(\vec{R} \cup \hat{\Pi}^{s}\left(z^{k}\right)\right) ; \overrightarrow{z^{k}}\right): z \preceq_{G L} z^{\prime}$. Hence, for each distribution $z$ we have to check whether there exists such $z^{\prime}$. Using Corollary 2 we can define each point $z^{\prime} \in C\left(\left(\vec{R} \cup \hat{\Pi}^{s}\left(z^{k}\right)\right) ; \overrightarrow{z^{k}}\right)$ using the equation $z^{\prime}=\overrightarrow{z^{k}}+\sum_{i=1}^{k-1} \mu_{i}\left(\overrightarrow{z^{k}}-\overrightarrow{z^{i}}\right)+\sum_{j=1}^{p} \beta_{j}\left(\overrightarrow{z^{k}}-\Pi^{j}\left(\overrightarrow{z^{k}}\right)\right)$ for $j: \Pi^{j}\left(\overrightarrow{z^{k}}\right)$ is obtained by swapping two consecutive elements in $\overrightarrow{z^{k}}$. Let the set of such indices $j$ be $J$; note that $|J|=p-1$.
We are going to make use of the following Theorem in our models.

Theorem 9 (Ogryczak \& Śliwiński, 2003). For any $z \in \mathbb{R}^{p}, \sum_{j=1}^{n} \overrightarrow{z_{j}}$ is the optimal value of the following LP problem:

\section{MODCUM (Model Cumulative)}

$\sum_{j=1}^{n} \overrightarrow{z_{j}}=\max n r_{n}-\sum_{h=1}^{p} d_{n h}$

subject to

$r_{n}-d_{n h}-z_{h} \leq 0$ for $h=1, \ldots, p$

$d_{n h} \geq 0 \quad$ for $h=1, \ldots, p$

The (alternative) optimal values of $r_{n}$ and $d_{n h}$ are as follows (see Ogryczak and Śliwiński, 2003 for details) $r_{n}^{*}=\vec{z}_{n}$ $+c$, where $c \geq 0$ is a constant and $d_{n h}^{*}=0$ for $h: z_{h}>\vec{z}_{n}$ and $d_{n h}^{*}=\vec{z}_{n}-z_{h}+c$ for $h: z_{h} \leq \vec{z}_{n}$. Hence at optimality we have $n r_{n}^{*}-\sum_{h=1}^{p} d_{n h}^{*}=n \vec{z}_{n}+n c-\sum_{h: z_{h} \leq \vec{z}_{n}}\left(\vec{z}_{n}+c-z_{h}\right)=$ $n \vec{z}_{n}-\sum_{h: z_{h} \leq \vec{z}_{n}}\left(\vec{z}_{n}-z_{h}\right)=\sum_{j=1}^{n} \vec{z}_{j}$. To illustrate, suppose that we have $z=(4,2,5)$ and we would like to know $\sum_{j=1}^{2} \overrightarrow{z_{j}}$. One optimal solution of this model is $r_{2}^{*}=4, d_{11}^{*}=0, d_{12}^{*}=4-2=2$, and $d_{13}^{*}=0$. Hence $\sum_{j=1}^{2} \overrightarrow{z_{j}}=2 * 4-(2+0+0)=6$. Note that at optimality we have $n-1 d_{n h}$ variables which are positive.

The following model will be used for checking whether $z$ is in $L$

$\left(L P_{1}\right)$

$\max \sum_{n=1}^{p} n r_{n}-\sum_{n=1}^{p} \sum_{h=1}^{p} d_{n h}$

subject to

$z_{h}^{\prime}-\sum_{i=1}^{k-1} \mu_{i}\left(\overrightarrow{z_{h}^{k}}-\overrightarrow{z_{h}^{i}}\right)-\sum_{j \in J} \beta_{j}\left(\overrightarrow{z_{h}^{k}}-\Pi^{j}\left(\overrightarrow{z^{k}}\right)_{h}\right)=\overrightarrow{z_{h}^{k}}$

for $h=1, \ldots, p$

$n r_{n}-\sum_{h=1}^{p} d_{n h} \geq \sum_{h=1}^{n} \vec{z}_{h}$ for $n=1, \ldots, p$

$r_{n}-d_{n h}-z_{h}^{\prime} \leq 0$ for $h, n=1, \ldots, p$

$\mu_{i} \geq 0$ for $i=1, \ldots, k-1$

$\beta_{j} \geq 0$ for $j=1, \ldots, p-1$

$d_{n h} \geq 0 \quad$ for $h, n=1, \ldots, p$

This model checks whether there exists $z^{\prime} \in C((\vec{R} \cup$ $\left.\left.\hat{\Pi}^{s}\left(z^{k}\right)\right) ; \overrightarrow{z^{k}}\right)$ such that $\sum_{j=1}^{n} \overrightarrow{z_{j}} \leq \sum_{j=1}^{n} \overrightarrow{z_{j}^{\prime}} \forall n=1, \ldots, p$. Constraint sets B.1 and B.2 ensure that $z^{\prime} \in C\left(\left(\vec{R} \cup \hat{\Pi}^{s}\left(z^{k}\right)\right) ; \overrightarrow{z^{k}}\right)$ and $\sum_{j=1}^{n} \overrightarrow{z_{j}} \leq \sum_{j=1}^{n} \overrightarrow{z_{j}^{\prime}} \forall n$, respectively. The objective function and constraint sets B.3 and B.6 are used to ensure that $\sum_{j=1}^{n} \vec{z}_{j}^{\prime}=n r_{n}^{*}-\sum_{h=1}^{p} d_{n h}^{*}$, where $r_{n}^{*}$ and $d_{n h}^{*}$ are the optimal values of these decision variables based on Theorem 9 .

To see what is happening intuitively, imagine deleting constraint set B.1 and fixing $z^{\prime}$. Then clearly the program will find the $\sum_{j=1}^{n} \overrightarrow{z_{j}^{\prime}} \forall n$ (because it can be decomposed $n$ subproblems of type MODCUM for each $n$, each of which will return $\sum_{j=1}^{n} \overrightarrow{z_{j}^{\prime}}$ ). Now we add back in constraint set B.1-so we are selecting a $z^{\prime}$ from the 
cone-but by the same reasoning whatever $z^{\prime}$ we select will be cumulative ordered.

Remark 2. At optimality, for each $n, n r_{n}^{*}-\sum_{h=1}^{p} d_{n h}^{*}=\sum_{j=1}^{n} \overrightarrow{z_{j}^{\prime}}$.

Proof of Remark 2. Suppose that this does not hold and we have $n r_{n}^{*}-\sum_{h=1}^{p} d_{n h}^{*}<\sum_{j=1}^{n} \vec{z}_{j}^{\prime}$. Then by setting $r_{n}^{* *}=\vec{z}_{n}^{\prime}$ and $d_{n h}^{* *}=0$ for $h: z_{h}^{\prime}>\vec{z}_{n}^{\prime}$ and $d_{n h}^{* *}=\vec{z}_{n}^{\prime}-z_{h}^{\prime}$ for $h: z_{h}^{\prime} \leq \vec{z}_{n}^{\prime}$, we can obtain $n r_{n}^{* *}-\sum_{h=1}^{p} d_{n h}^{* *}=\sum_{j=1}^{n} \overrightarrow{z_{j}^{\prime}}$ while satisfying all constraints. This contradicts the optimality of $\left(r_{n}^{*}, d_{n h}^{*}\right)$.

Suppose that we have $n r_{n}^{*}-\sum_{h=1}^{p} d_{n h}^{*}>\sum_{j=1}^{n} \overrightarrow{z_{j}^{\prime}}$. We can show that such a solution is not feasible as constraint set B.3 $\left(r_{n}-\right.$ $d_{n h}-z_{h}^{\prime} \leq 0$ forh $\left.=1, \ldots, p\right)$ ensures that $r_{n}^{*}-d_{n h}^{*} \leq \vec{z}_{h}^{\prime}$ for all $h=$ $1, \ldots, p$. For any $n$, consider the corresponding constraints having right hand side $z_{h}^{\prime}: z_{h}^{\prime}=\vec{z}_{i}^{\prime}: i \leq n$. Aggregating these $n$ constraints would give $n r_{n}^{*}-\sum_{h: z_{h}^{\prime}=\vec{z}_{i}^{\prime}: i \leq n} d_{n h}^{*} \leq \sum_{i=1}^{n} \vec{z}_{i}^{\prime}$. Hence $n r_{n}^{*}-$ $\sum_{h=1}^{p} d_{n h}^{*} \leq n r_{n}^{*}-\sum_{h: z_{h}^{\prime}=\vec{z}_{i}^{\prime}: i \leq n} d_{n h}^{*} \leq \sum_{i=1}^{n} \vec{z}_{i}^{\prime}=\sum_{j=1}^{n} \vec{z}_{j}^{\prime}$.

Note that it is also possible to use a feasibility problem here: One can check whether there exist any feasible $r_{n}$ and $d_{n h}$ values by changing the objective function as max 0 . If there exist any feasible $r_{n}$ and $d_{n h}$ values (leading to solution that satisfies all the relevant constraints B.2, B.3 and B.6), then the optimal values of $r_{n}^{*}$ and $d_{n h}^{*}$ in $L P_{1}$ (which maximise $\sum_{n=1}^{p} n r_{n}-\sum_{n=1}^{p} \sum_{h=1}^{p} d_{n h}$ ) also satisfy the constraint set B.2. This guarantees that constraint set B.2 is satisfied hence $\sum_{j=1}^{n} \overrightarrow{z_{j}} \leq \sum_{j=1}^{n} \overrightarrow{z_{j}^{\prime}} \forall n$. We use max 0 as the objective function of $L P_{1}$. If the problem is feasible then $z \in \hat{L}\left(z^{k}\right)$ hence $z \in L\left(z^{k}\right)$.

$L P_{1}$ is an $L P$ problem with $p^{2}+3 p+k-2$ variables and $p^{2}+2 p$ constraints excluding the set constraints (Recall that the we use $p-1$ permutations of the lower generator). Note that, we work on problems where we ask the SP to compare distributions, i.e. vectors. Requesting the SP to compare vectors is only meaningful when the size of the vectors $(p)$ is reasonable. $p$ must be in any case quite small as SPs will not be able to compare highly dimensional distributions.

Also note that in the algorithms which use two-point cones only a simpler LP can be used as explained in the next subsection.

B.2. Checking whether a given point is in $L$ when using two-point cones

In order to check whether $z \in L\left(z^{k}\right)$, we check whether there exists a point $z^{\prime} \in C\left(\overrightarrow{z^{1}} ; \overrightarrow{z^{2}}\right): z \preceq_{G L} z^{\prime}$ for any two generators $z^{1}$ and $z^{2}$. We use the following LP model:

$\left(L P_{3}\right)$

Max 0

subject to

$z_{h}^{\prime}-\mu\left(\overrightarrow{z_{h}^{2}}-\overrightarrow{z_{h}^{1}}\right)=\overrightarrow{z_{h}^{2}} \quad$ for $h=1, \ldots, p$

$n r_{n}-\sum_{h=1}^{p} d_{n h} \geq \sum_{j=1}^{n} \vec{z}_{j}$ for $n=1, \ldots, p$

$r_{n}-d_{n h}-z_{h}^{\prime} \leq 0$ for $h, n=1, \ldots, p$

$\mu \geq 0$

$d_{n i} \geq 0$ for $i, n=1, \ldots, p$
This model checks whether there exists $z^{\prime} \in C\left(\overrightarrow{z^{1}} ; \overrightarrow{z^{2}}\right)$ such that $\sum_{j=1}^{n} \overrightarrow{z_{j}} \leq \sum_{j=1}^{n} \overrightarrow{z_{j}^{\prime}} \forall n$. Constraint sets B.7 and B.8 ensure that $z^{\prime} \in$ $C\left(\overrightarrow{z^{1}} ; \overrightarrow{z^{2}}\right)$ and $\sum_{j=1}^{n} \overrightarrow{z_{j}} \leq \sum_{j=1}^{n} \overrightarrow{z_{j}^{\prime}} \forall n$, respectively. Constraint set B.9 is used to ensure that $\sum_{h=1}^{n}{\overrightarrow{z^{\prime}}}_{h}=n r_{n}^{*}-\sum_{h=1}^{p} d_{n h}^{*}$, where $r_{n}^{*}$ and $d_{n h}^{*}$ are the optimal values of these decision variables based on Theorem 9.

This is an LP problem with $p^{2}+2 p+1$ variables and $p^{2}+2 p$ constraints excluding the set constraints.

\section{B.3. Checking whether a given distribution is in $U$}

If $z \in U\left(z^{k}\right)$ then there exists $z^{\prime} \in P\left(\vec{R} ; \overrightarrow{z^{k}}\right): z^{\prime} \preceq_{G L} z$. The following model will be used for the corresponding check:

$\operatorname{Max} \epsilon$

subject to

$z_{h}^{\prime}-\mu \overrightarrow{z^{k}}{ }_{h}-\sum_{i=1}^{k-1} \mu_{i}\left(\vec{z}_{h}^{i}\right)=0$ for $h=1, \ldots, p$

$\sum_{h=1}^{n} z_{h}^{\prime}+\epsilon \leq \sum_{h=1}^{n} \vec{z}_{h}$ for $n=1, \ldots, p$

$\sum_{i=1}^{k-1} \mu_{i}+\mu=1$

$\mu_{i} \geq 0 \forall i$

$\mu \geq 0$

Constraint sets B.12 and B.14 ensure that $z^{\prime} \in P\left(\vec{R} ; \overrightarrow{z^{k}}\right)$ and constraint set B.13 ensures that $z^{\prime} \preceq_{G L} \quad z$ by ensuring $\sum_{j=1}^{n} \overrightarrow{z_{j}^{\prime}} \leq$ $\sum_{j=1}^{n} \overrightarrow{z_{j}} \forall n$. If at optimality, $\epsilon^{*} \geq 0$ then $z \in U\left(z^{k}\right)$.

It is an LP problem with $p+k+1$ variables and $2 p+1$ constraints excluding the set constraints.

\section{Appendix C. Explanation of the subroutines}

Dominancecheck checks generalised Lorenz dominance. We store the information on generalised Lorenz dominance relations in an $n^{*} n$ matrix called Dominancemat. Dominancemat $(i, j)=1$ if distribution $i$ generalised Lorenz dominates distribution $j$; 0 otherwise.

Getinfo subroutine gathers information from the SP by providing him with a set of distributions. The preference information gathered is in form of ranking of the distributions in the sample. The size of this set is controlled by a parameter called samplesize. We set samplesize $=2$, hence use pairwise comparisons in our experiments. But it is possible to use samples with more than two distributions, in which case the SP is asked to rank these distributions from the best to the worst.

The distributions to be put to the SP are selected according to a predetermined rule. In the first iteration we rank the distributions according to their Euclidean distances to an ideal point (IP) whose coordinates are defined as follows:

$I P_{i}=\operatorname{Max}_{z \in Z} \sum_{j=1}^{i} \overrightarrow{z_{j}} \forall i=1, \ldots, p$. IP.
We select the ones having the least (Euclidean) distances to the 
In the following iterations, we select the distributions on whose ranks we have the least information. Before asking the SP we perform a consistency check for the pair as follows: When we determine a pair of distributions $(a, b)$ to be presented to the SP, we perform a consistency check to see whether any of the following two cases would lead to violation of the consistency assumption given the preference information so far: $a \succ b$ or $b \succ a$. Consistency would be violated if, for example $a$ is c-dominated by a cone with a lower generator $b$ (which should imply that $b>a$ ) and when asked, the SP indicates that he prefers $a$ over $b$. If only one of these cases $(a \succ b$ or $b \succ a$ ) would allow consistency, then we choose it without asking the SP. If both cases are possible (none would violate consistency) we ask the SP. If for a pair $(a, b)$ neither $a \succ b$ nor $b \succ a$ would allow consistency then the SP is inconsistent.

For example, suppose that the SP has provided the following preference information so far (about 10 distributions indexed from $z^{1}$ to $\left.z^{10}\right): z^{1} \succ z^{2}, z^{4} \succ z^{7}, z^{9} \succ z^{3}, z^{7} \succ z^{2}$. Hence we have the following quasi-orderings: $z^{1} \succ z^{2}$ and $z^{4} \succ z^{7} \succ z^{2}$, and $z^{9} \succ z^{3}$. Suppose that we ask the SP about $\left(z^{1}, z^{3}\right)$. If $z^{3} \succ z^{1}$ and $z^{4} \in L\left(z^{1}\right)$ (as $\exists z \in C\left(z^{3} ; z^{1}\right): z^{4} \preceq_{G L} z$ ) the quasiordering will become $z^{9} \succ z^{3}$ $\succ z^{1} \succcurlyeq z^{4} \succ z^{7} \succ z^{2}$. Moreover, suppose that $z^{9} \in L\left(z^{2}\right)$ (i.e., $\exists z \in C\left(z^{3}\right.$, $\left.\left.z^{1} ; z^{2}\right): z^{9} \preceq_{G L} z\right)$ resulting in $z^{9} \succ z^{3} \succ z^{1} \succcurlyeq z^{4} \succ z^{7} \succ z^{2} \succcurlyeq z^{9}$, which results in inconsistency. We try to avoid such situations as much as possible by checking whether such cases occur before asking about a pair. In our example case, we also check whether $z^{1} \succ z^{3}$ would lead to inconsistency and if not we do not ask the SP but take $z^{1} \succ z^{3}$. If both cases $\left(z^{1} \succ z^{3}\right.$ and $\left.z^{3} \succ z^{1}\right)$ lead to inconsistency we stop the procedure and inform the SP about the situation.

We keep track of the information on a distribution's possible ranks using an $n^{*} 2$ matrix called boundmatrix. In this matrix each row is dedicated to a distribution and the two values in each row show the minimum and maximum possible ranks of the distribution, respectively. At the beginning these values are set to 1 and $n$ for all the distributions. Whenever new information is available, this matrix is updated accordingly. While asking the SP for preference information, we choose the distributions for which the difference between the maximum and minimum possible rank is larger.

When preference information is obtained, it is stored in an $n^{*} n$ matrix called Userpreference. This matrix keeps the information for cone and polyhedron generation. When new information is gathered, the Userpreference matrix is updated based on transitivity. For example, if from previous iterations we know that distribution $i$ is preferred to distribution $j$ and in the current iteration we are given $j$ is preferred to distribution $k$, then we update the matrix setting Userpreference $(i, k)=1$. This allows us to generate the largest cone for a given lower generator.

Conegeneration performs the checks related to the cones (i.e., lower set) and polyhedra (i.e., upper set). The two LPs, $L P_{1}$ and $L P_{2}$, are generated and solved in this subroutine. Whenever possible, redundant cones/polyhedra and checks are avoided. We do not solve the LPs for a distribution if we already know that it is cdominated by/c-dominates the cone's lower generator (recall that we check consistency before asking the question hence inconsistency does not arise). Since some of the new information obtained through these checks leads us to new cones and polyhedra, we repeat this subroutine until there is no useful new information. We check this condition by using a binary variable called newinfo.

Countassigned Recall that we keep the lowest and highest possible ranks for an distribution in boundmatrix. At the end of each iteration, for each distribution, we count the distributions that it c-dominates/is preferred to and the ones that it is c-dominated by/less preferred than. We update the information on boundmatrix accordingly. We then count the number of distributions whose rank we know, i.e., whose maximum and minimum possible ranks are equal. This information is then used to decide whether to terminate the algorithm.

\section{References}

Argyris, N., Morton, A., \& Figueira, J. R. (2014). CUT: A multi-criteria approach for concavifiable preferences. Operations Research, 62(3), 633-642.

Armbruster, B., \& Delage, E. (2015). Decision making under uncertainty when preference information is incomplete. Management Science, 61(1), 111-128.

Atkinson, A. B. (1970). On the measurement of inequality. Journal of Economic Theory, 2, 244-263.

Baatar, D., \& Wiecek, M. (2006). Advancing equitability in multiobjective programming. Computers \& Mathematics with Applications, 52(1-2), 225-234.

Culyer, A. J. (1989). The normative economics of health care finance and provision. Oxford Review of Economic Policy, 5(1), 34-58.

Dasgupta, P., Sen, A., \& Starrett, D. (1973). Notes on the measurement of inequality. Journal of Economic Theory, 6, 180-187.

Diamond, P. A. (1967). Cardinal welfare, individualistic ethics, and interpersonal comparison of utility: Comment. The Journal of Political Economy, 75(5), 765.

Gravel, N., \& Moyes, P. (2013). Utilitarianism or welfarism: does it make a difference? Social Choice and Welfare, 40(2), 529-551.

Hardy, G. H., Littlewood, J. E., \& Polya, G. (1934). Inequalities. Cambridge.

Hazen, G. (1983). Preference convex unanimity in multiple criteria decision making. Mathematics of Operations Research, 8(4), 505-516.

Hurley, S. L. (1992). Natural reasons: Personality and polity. Oxford University Press on Demand.

Jeffrey, R. (1982). The sure thing principle. PSA: Proceedings of the Biennial Meeting of the Philosophy of Science Association, 1982, 719-730.

Karsu, Ö. (2013). Using holistic multicriteria assessments: The convex cones approach. In Wiley encyclopedia of operations research and management science (pp. 1-14)

Karsu, Ö. (2016). Approaches for inequity-averse sorting. Computers \& OR, 66, 67-80

Karsu, Ö., \& Azizoglu, M. (2012). The multi-resource agent bottleneck generalized assignment problem. International Journal of Production Research, 50(2), 309-324.

Karsu, Ö., \& AzizogĨu, M. (2014). Bicriteria multiresource generalized assignment problem. Naval Research Logistics (NRL), 61(8), 621-636.

Karsu, Ö., \& Morton, A. (2014). Incorporating balance concerns in resource allocation decisions: A bi-criteria modelling approach. Omega, 44, 70-82.

Karsu, Ö., \& Morton, A. (2015). Inequity averse optimization in operational research. European Journal of Operational Research, 245(2), 343-359.

Korhonen, P., Wallenius, J., \& Zionts, S. (1984). Solving the discrete multiple criteria problem using convex cones. Management Science, 30(11), 1336-1345.

Kostreva, M. M., \& Ogryczak, W. (1999). Linear optimization with multiple equitable criteria. RAIRO Operations Research, 33, 275-297.

Kostreva, M. M. Ogryczak, W. \& Wierzbicki, A. (2004). Equitable aggregations and multiple criteria analysis. European Journal of Operational Research, 158(2), 362-377.

Levy, H. (1992). Stochastic dominance and expected utility: Survey and analysis. Management Science, 38(4), 555-593.

Lorenz, M. O. (1905). Methods of measuring the concentration of wealth. Publications of the American Statistical Association, 9(70), 209-219.

Luss, H. (2010). Equitable bandwidth allocation in content distribution networks. Naval Research Logistics, 57(3), 266-278.

Luss, H. (2012). Equitable resource allocation: Models, algorithms and applications. John Wiley \& Sons, Inc.

Marshall, A., Olkin, I., \& Arnold, B. (2009). Inequalities: Theory of majorization and its applications. Springer Series in Statistics (2nd). New York, USA: Springer.

Morton, A. (2014). Aversion to health inequalities in healthcare prioritisation: A multicriteria optimisation perspective. Journal of Health Economics, 36, 164-173.

Müller, A., \& Stoyan, D. (2002). Comparison methods for stochastic models and risks (pp. pp.31-37). Wiley.

Mut, M., \& Wiecek, M. M. (2011). Generalized equitable preference in multiobjective programming. European Journal of Operational Research, 212(3), 535-551.

Ogryczak, W. (1999). On the distribution approach to location problems. Computers E Industrial Engineering, 37(3), 595-612.

Ogryczak, W. (2000). Inequality measures and equitable approaches to location problems. European Journal of Operational Research, 122, 374-391.

Ogryczak, W. (2009). Inequality measures and equitable locations. Annals of Operations Research, 167(1), 61-86

Ogryczak, W., \& Śliwiński, T. (2003). On solving linear programs with the ordered weighted averaging objective. European Journal of Operational Research, 148(1), $80-91$

Ogryczak, W., Wierzbicki, A., \& Milewski, M. (2008). A multi-criteria approach to fair and efficient bandwidth allocation. Omega, 36(3), 451-463.

Ok, E. (1998). Inequality averse collective choice. Journal of Mathematical Economics, $30,301-321$

Rothschild, M., \& Stiglitz, J. H. (1973). Some further results on the measurement of inequality. Journal of Economic Theory, 6(2), 188-204.

Roy, B. (1971). Problems and methods with multiple objective functions. Mathematical Programming, (1), 239-266.

Savage, L. (1954). The foundations of statistics. Wiley publications in statistics. John Wiley and Sons.

Sen, A., \& Foster, J. (1997). On economic inequality. Oxford: Clarendon Press. Expanded edition 
Shaked, M., \& Shanthikumar, J. G. (2007). Stochastic orders (pp. 59-65). Springer Science \& Business Media.

Shorrocks, A. (1983). Ranking income distributions. Economica, 50, 3-17.

Thistle, P. D. (1989). Ranking distributions with generalized lorenz curves. Southern Economic Journal, 56(1), pp.1-12.

Wakker, P. (1989). Additive representation of preferences: A new foundation of decision analysis. Kluwer Academic Publishers.
WB (2011). Available at http://data.worldbank.org/indicator/SI.DST.05TH.20 (Accessed on 15 August 2011).

WIDER (2011). Available at http://www.wider.unu.edu/research/Database/en-GB wiid (Accessed on 15 August 2011).

Young, P. H. (1994). Equity in theory and practice (1st). Princeton: Princeton University Press. 